\title{
Injection with a single dipole kicker into the MAX IV storage rings
}

\author{
S.C. Leemann* \\ MAX-lab, Lund University, S-22363 Lund, Sweden
}

\section{A R T I C L E I N F O}

\section{Article history:}

Received 14 May 2012

Received in revised form

11 July 2012

Accepted 11 July 2012

Available online 22 July 2012

Keywords:

Synchrotron light source

Storage ring

Injection

Pulsed magnet

Dipole kicker

\begin{abstract}
A B S T R A C T
Injection into the two MAX IV storage rings will not make use of a 4-kicker local injection bump. Instead, pulsed multipole injection will be used for initial filling as well as top-up injection. Since commissioning a pulsed multipole magnet for injection into a storage ring is non-trivial, it has been decided to install a single dipole kicker magnet into the storage rings to provide a simple method for injection during early commissioning. Design studies have revealed that injection with a single dipole kicker into the MAX IV storage rings is not only efficient, but also allows for accumulation of beam. Although this accumulation cannot be made transparent to users (i.e. it is not compatible with user topup operation), it does provide a simple and robust injection method during commissioning. In addition, the dipole kicker can be used as a pinger magnet during machine studies with a single-bunch filling. This paper reports on the design studies performed for dipole kicker injection into the MAX IV storage rings and presents a summary of the expected performance of such an injection scheme.
\end{abstract}

(c) 2012 Elsevier B.V. All rights reserved.

\section{Introduction}

The MAX IV facility [1] which is currently under construction comprises two storage rings and a short-pulse facility driven by a $3.5 \mathrm{GeV}$ linac. Both the $3 \mathrm{GeV}$ storage ring $[2,3]$ and the $1.5 \mathrm{GeV}$ storage ring [4,5] will be operated in top-up mode with $500 \mathrm{~mA}$ stored current. Injection into these two rings is performed by the underground full-energy linac [6] via two vertical transfer lines. An overview of the facility layout is shown in Fig. 1. Originally, it was foreseen to inject into the storage rings using a closed fourkicker injection bump [7]; however, subsequent design studies showed that injection with a single pulsed multipole magnet offers significant advantages over the conventional local bump scheme [8]. Not only does pulsed multipole injection involve fewer magnets and relax synchronization and alignment requirements, it also has the potential to make top-up injection transparent to users despite the very hard stability tolerances of an ultra-low emittance light source like the MAX IV $3 \mathrm{GeV}$ storage ring. The MAX IV storage rings will be the first light sources to be designed and operated from the beginning with pulsed multipole injection.

Despite the clear advantages of pulsed multipole injection, commissioning a new storage ring with this type of injection scheme is non-trivial. The pulsed multipole magnet kick shows a strong dependance on the injection amplitude. This amplitude in turn is heavily influenced by both the linear and nonlinear optics

\footnotetext{
* Tel.: +46703873168 .

E-mail address: simon.leemann@maxlab.lu.se
}

as well as the exact position and angle of the beam at the injection point. Additionally, injected bunches have to be transported from the injection septum through a fairly long magnet section successfully before reaching the pulsed multipole where they can be captured within the storage ring acceptance. Since storage ring optics as well as injection positions and angles are initially not exactly known and are prone to errors due to misalignments, faulty cabling, or power supply malfunction in a just assembled storage ring, commissioning a new storage ring with only a pulsed multipole for injection is very demanding and entails significant risks. Optics and alignment errors can be diagnosed and corrected once small amounts of beam can be injected and followed around the storage ring. However, if beam cannot be injected into the storage ring at all or if the injected charge is so low that diagnostics do not deliver data at the required accuracy, discovery and removal of errors can become very complicated and time-consuming.

In order to reduce the risk of commissioning two new storage rings with only pulsed multipole injection, it was decided to install a single dipole kicker in each storage ring. Such a dipole kicker can be used to inject charge from the linac into the storage ring without large dependence on optics or alignment. In this way it becomes a robust injection method during early commissioning when neither the optics nor the injection orbit is well known and/ or corrected. This paper will describe how a scheme using only a single dipole kicker for injection into an ultra-low emittance storage ring can be designed including estimates of the injection efficiency and specifications for the required magnet and pulser. Details of the linac and transfer lines as well as top-up injection requirements have been reported in Ref. [8] and will not be 


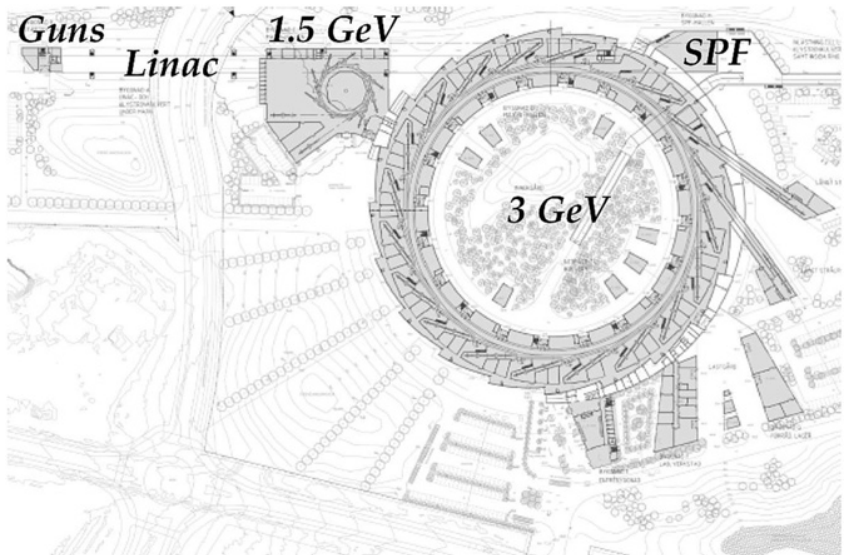

Fig. 1. Layout of the MAX IV facility as seen from above. The guns, linac, and short-pulse facility (SPF) are underground. The $1.5 \mathrm{GeV}$ and $3 \mathrm{GeV}$ storage rings are above ground. Two vertical transfer lines connect the linac extraction points with the storage ring injection points.

repeated here. The next section describes how an injection scheme with only a single dipole kicker is designed. The two following sections present the actual solutions and the expected performance for the two storage rings of the MAX IV facility. The final section discusses technical issues and gives specifications for the dipole kicker magnets, chambers, and pulsers. A description of the use of these kickers as horizontal pinger magnets concludes the paper.

\section{Injection with a single dipole kicker}

Bunches are injected from the linac through an achromatic vertical transfer line into the storage rings where they reach the injection point (IP), i.e. the magnetic end of the vertical injection septum, with an injection amplitude and angle $\left(x_{\text {inj }}, x_{\text {inj }}^{\prime}\right)$ in the horizontal plane defined by the stored beam. The injection invariant $A_{\text {inj }}$ of these bunches is then given by $[9,10]$

$A_{\text {inj }}^{2}=\gamma_{\text {inj }} x_{\text {inj }}^{2}+2 \alpha_{\text {inj }} x_{\text {inj }} x_{\text {inj }}^{\prime}+\beta_{\text {inj }} x_{\text {inj }}^{\prime 2}$

where the Twiss parameters $\alpha, \beta$, and $\gamma$ have been evaluated at the IP. These bunches are transported to the dipole injection kicker (KI) where their position and angle are given by $\left(x_{\mathrm{ki}}, x_{\mathrm{ki}}^{\prime}\right)$. At this point the dipole kicker kicks the particles by $\theta_{\mathrm{ki}}$ thus reducing the angle

$x_{\mathrm{ki}}^{\prime} \longrightarrow x_{\mathrm{red}}^{\prime}=x_{\mathrm{ki}}^{\prime}+\theta_{\mathrm{ki}}$

and the invariant to $A_{\text {red }}$. In order for injection with a single dipole kicker to be successful, the reduced invariant must obviously lie within the storage ring's horizontal acceptance, i.e.

$A_{\text {red }}<A_{x}$.

A reduced invariant of zero can be achieved if a cross-over location (i.e. $x_{\mathrm{ki}}=0$ ) is chosen and a sufficiently strong dipole kicker is available (i.e. $\theta_{\mathrm{ki}}=-x_{\mathrm{ki}}^{\prime}$ ). Such a cross-over occurs at a phase advance

$\Psi=\left(n-\frac{1}{2}\right) \pi-\phi_{\text {inj }}, \quad n=1,2,3, \ldots$

from the dipole kicker, where $\phi_{\text {inj }}$ is the phase advance between the symmetry point (e.g. center of the injection straight) and the IP. At such a cross-over a dipole kick of

$\theta_{\mathrm{ki}}=\frac{A_{\mathrm{inj}}}{\sqrt{\beta_{\mathrm{ki}}}}$

then leads to zero reduced invariant. It is important to note, however, that although applying such a dipole kick will lead to zero reduced invariant of the injected bunch, any beam already stored in the machine will be kicked out, i.e., such a kick does not allow for accumulation of beam.

Since it is not always possible to install the dipole kicker in a cross-over location, a non-zero reduced invariant will remain; however, it can be minimized by adjusting the dipole kick properly. For a dipole kicker installed at an arbitrary phase $\phi_{\mathrm{ki}}$ from the symmetry point, the minimum reduced invariant is achieved with the dipole kick

$\theta_{\mathrm{ki}}=\frac{A_{\mathrm{inj}}}{\sqrt{\beta_{\mathrm{ki}}}}\left|\sin \phi_{\mathrm{ki}}\right|$.

Of course the above reasoning and analytical solutions are only valid under the assumption of linear betatron motion. In real storage rings betatron motion is nonlinear, especially in ultra-low emittance storage ring such as the MAX IV $3 \mathrm{GeV}$ storage ring with its strong sextupoles and octupoles [11]. In such storage rings the single-dipole kicker injection scheme has to be derived from tracking. Tracking studies reveal the actual trajectory of the injected bunch and indicate candidate locations for the dipole kicker, i.e., locations with low amplitudes and angles sufficiently low so that the dipole kicker can kick bunches into the storage ring acceptance. In addition to the desired optics, these locations need to offer sufficient space for the kicker magnet and should ideally be as close to the IP as possible. In this way the likelihood that the injected bunches can initially be transported from the IP to the dipole kicker increases. The choice of location for the dipole kicker in the MAX IV storage rings will be described on the two following sections.

Once a location for the dipole kicker has been determined, an ideal kick strength $\theta_{\mathrm{ki}}$ to minimize the resulting invariant $A_{\text {red }}$ can be calculated. Two other strengths are also revealed by tracking studies: the maximum kick $\theta_{\max }$ a centered beam (e.g. the stored beam) can receive before it is ejected from the storage ring's acceptance $A_{x}$, and the minimum kick $\theta_{\min }$ required to kick the injected beam into the storage ring acceptance, i.e., the kick that leads to the reduced invariant $A_{\text {red }}=A_{x}$. If these two kick strengths open up a window, i.e., $\theta_{\min }<\theta_{\max }$, there exists a dipole kicker setting where injected bunches can be captured, while already captured particles are not ejected from the storage ring. With such a dipole kicker setting, this single dipole kicker can be used to accumulate charge in the storage ring as long as there is sufficient time between two injections for the injected particles to damp down to the stored beam.

For a given location of the dipole kicker it is sometimes possible to create a cross-over at the dipole by slightly modifying the beam position and/or angle at the IP. While this can require increasing the injection invariant, if a cross-over is created at the dipole kicker and enough dipole kick strength is available (this can be more than the nominally required strength, especially in cases where the injection invariant is increased by the offset at the IP), the injected beam's position and angle can both be reduced to zero immediately after the injection dipole, hence minimizing the reduced invariant. During early commissioning when more sever misalignments may be present and/or optics are not set to their design values, this is an interesting injection option because it reduces requirements for available physical aperture to a minimum and increases tolerance to erroneous optics. If an injected beam can be created so that the reduced invariant becomes zero or near-zero after the dipole kicker, chances are increased that the beam can be threaded around the machine to find the closed orbit which is necessary in order to start reducing misalignments and finding optics errors.

The following two sections will present solutions for injection with a single dipole kicker into the MAX IV storage rings where the above-mentioned tracking campaign has been followed to 
design injection schemes and quantify their injection efficiency. In addition to the nominal injection settings, tolerances for injection as well as schemes for on-axis injection and beam accumulation shall be derived.

\section{Injection into the $3 \mathrm{GeV}$ storage ring}

The IP in the $3 \mathrm{GeV}$ storage ring is the magnetic end of the $5^{\circ}$ injection septum $(1902 \mathrm{~mm}$ downstream of the center of the injection straight or $444 \mathrm{~mm}$ upstream of the center of the BPM at the beginning of the first achromat). Fig. 2 shows a schematic of the injection layout. The optics in the horizontal plane at the IP are $\beta_{\text {inj }}=9.402 \mathrm{~m}$ and $\alpha_{\mathrm{inj}}=-0.211$. The lateral placement of the septum was chosen so that the blade becomes the limiting horizontal aperture of the storage ring $A_{x}^{2}=11.11 \mathrm{~mm}$ mrad while not limiting acceptance more than necessary.

Fig. 3 (top) shows the amplitude of the injected bunch from the IP to the dipole kicker and through the first achromat. Bunches are injected at the IP at $x_{\text {inj }}=-13.5 \mathrm{~mm}$ parallel to the stored beam which gives an injected invariant of $A_{\mathrm{inj}}^{2}=20.25 \mathrm{~mm}$ mrad. The dipole kicker is installed in the first short straight section downstream of the injection straight. This is not only close to a cross-over location, but it also leaves only $3 \mathrm{~m}$ and a limited number of magnets between the IP and the dipole kicker. This increases the probability that injection into the ring can be carried out in the early stages of commissioning when severe misalignments and erroneous optics cannot be entirely ruled out. Once beam can be injected into the ring, diagnosing and resolving such errors become much easier. The exact position of the center of the dipole kicker $(\mathrm{KI})$ is in the first short straight, $473 \mathrm{~mm}$ downstream of the center of the sextupole SDend in the preceding matching cell (or $957 \mathrm{~mm}$ upstream of the center of the first quadrupole QFm is the following unit cell). At the location of $\mathrm{KI}$ the storage ring optics in the horizontal plane are $\beta_{\mathrm{ki}}=1.170 \mathrm{~m}$ and $\alpha_{\mathrm{ki}}=-0.694$ and the coordinates of the injected bunch are $x_{\mathrm{ki}}=$ $1.739 \mathrm{~mm}$ and $x_{\mathrm{ki}}^{\prime}=4.963 \mathrm{mrad}$. Tracking reveals that reducing the divergence of the injected bunch at KI by $x_{\mathrm{ki}}^{\prime}=4.963 \rightarrow 1.030 \mathrm{mrad}$, i.e. $\theta_{\mathrm{ki}}=3.933 \mathrm{mrad}$, minimizes the resulting reduced invariant at $A_{\text {red }}^{2}=2.56 \mathrm{~mm}$ mrad. The phase space plot in Fig. 3 (bottom) demonstrates clearly why the minimum invariant is reached with this kick.
It should be noted that the reduced invariant achieved in this way is not only well within the storage ring acceptance $A_{x}$, but it also comparable to what can be achieved with the pulsed
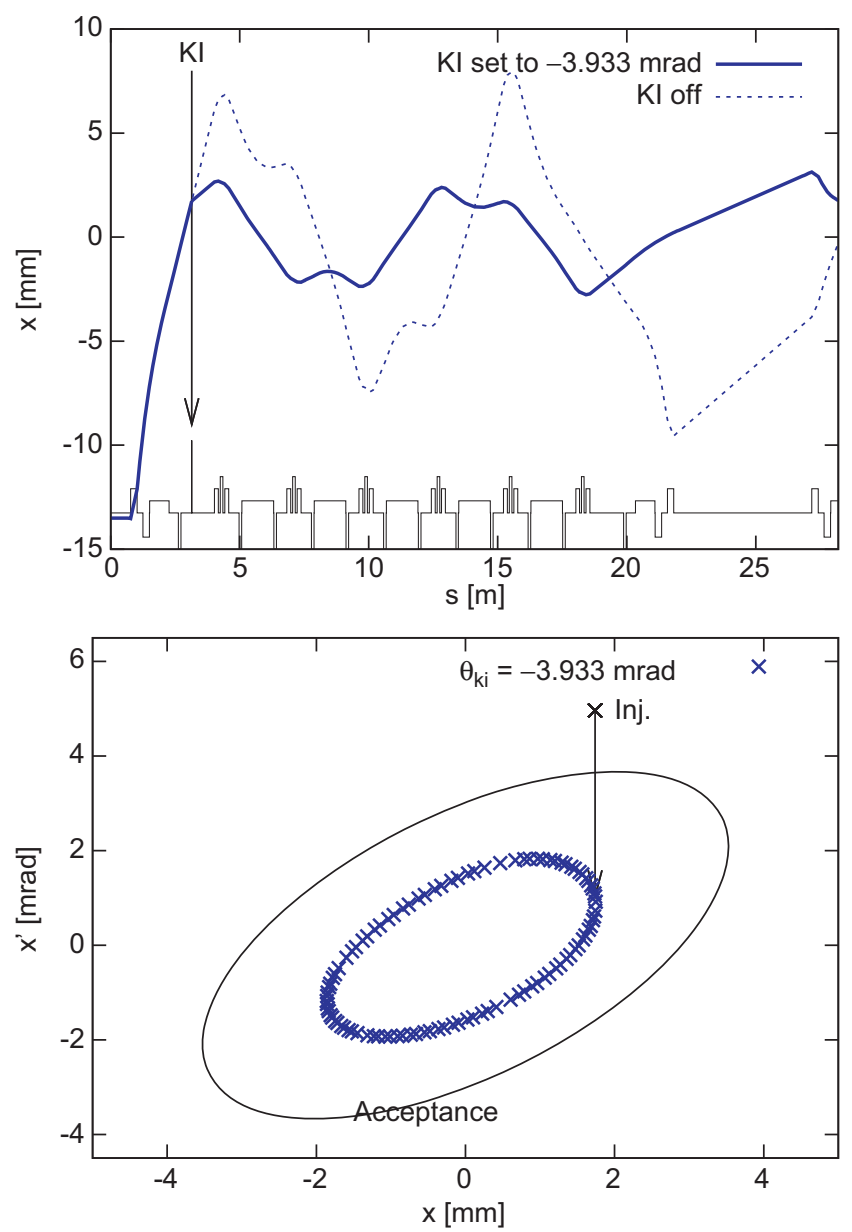

Fig. 3. Injection with the dipole kicker into the MAX IV $3 \mathrm{GeV}$ storage ring. Top: amplitude of the injected bunch from the IP through the first achromat and second long straight. Bottom: capture and the first 100 turns in the storage ring are shown at the location of the dipole kicker. As demonstrated by the plot, setting the dipole kicker to $\theta_{\mathrm{ki}}=-3.9 \mathrm{mrad}$ minimizes the reduced invariant.
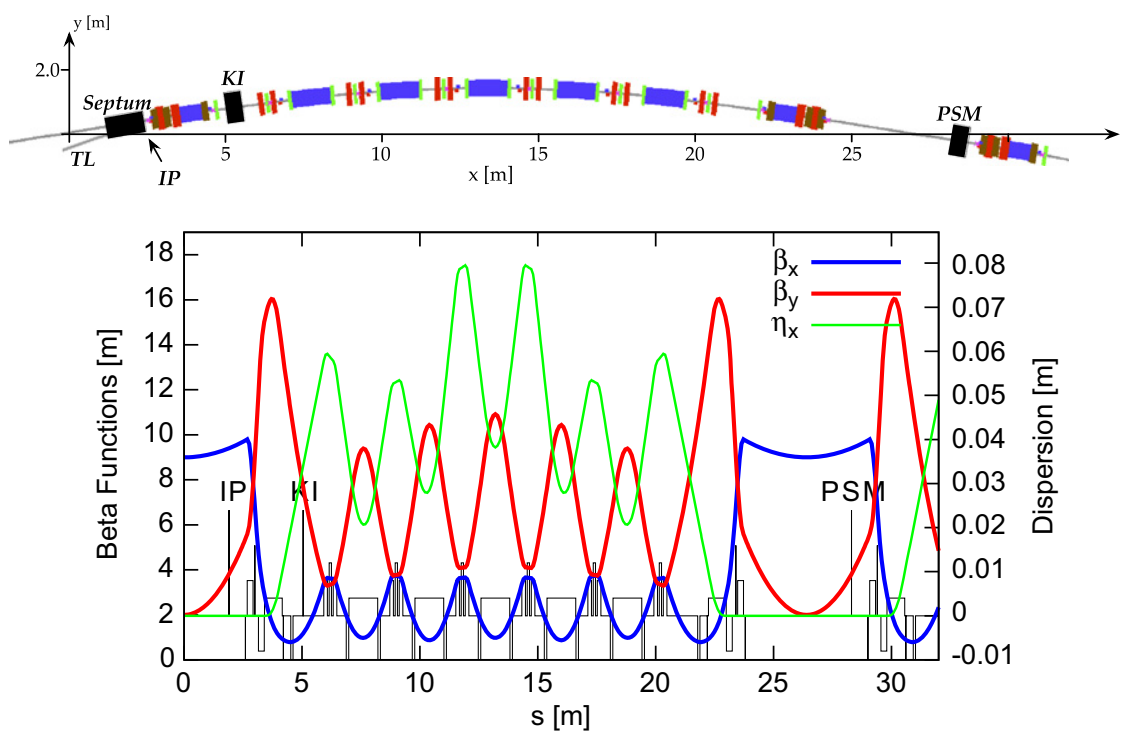

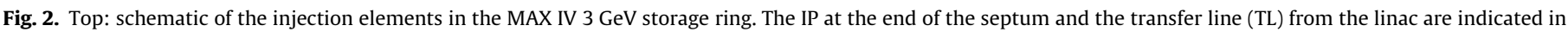

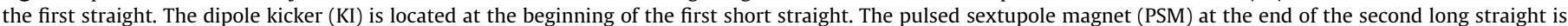
also displayed. Bottom: the optics in the storage ring for the area depicted in the schematic above. 
sextupole magnet injection scheme [8]. In order to assess injection efficiency when using the dipole kicker in this manner, an ensemble of particles has been tracked from the IP and through the storage ring lattice for its first five turns. The particle ensemble (a Gaussian particle distribution, $n=1000$, cut-off at $3 \sigma$ ) is modeled using the expected parameters after acceleration from the thermionic rf-gun in the linac and transport through the transfer line (a summary of this process is given in Ref. [8]). The normalized emittance of the injected bunch is assumed to be $\varepsilon_{n}=10 \mathrm{~mm}$ mrad (corresponding to $\varepsilon_{x}=1.7 \mathrm{~nm} \mathrm{rad}$ ), the energy spread is $\sigma_{\delta}=0.1 \%$. The transfer line optics are adjusted to deliver $\beta_{x}=13.286 \mathrm{~m}$ and $\alpha_{x}=-0.002$ at the IP [12]. The results of such a tracking study using DIMAD are shown in Fig. 4. Injection and capture in the storage ring are shown for the above detailed dipole kicker setting. The plot shows the data at the IP. The position of the septum is indicated. In this process no particles are lost which indicates injection efficiency should be very high.

\subsection{Tolerances}

During actual operation, the linac could have slightly different optics or the extracted bunch could suffer an emittance and/or energy spread increase. In order to assess tolerances for linac operation, two extreme cases are displayed in Fig. 5 where capture efficiency is no longer $100 \%$. A situation where the energy spread of the injected bunch has increased to $\sigma_{\delta}=1.8 \%$ (which is actually already beyond the energy acceptance of the transfer line) shows smearing out of the injected bunch and tails starting to scrape the acceptance. The case with a six-fold increased emittance of $\varepsilon_{n}=60 \mathrm{~mm}$ mrad simulates emittance blow-up in the linac as well as optics mismatches up to the IP. In this situation a blow-up of the bunch is observed, without significant distortion however. Any further increase of emittance will lead to particle loss on the septum blade.

\subsection{Accumulation}

As already detailed in the Introduction, the dipole kicker can be used for injection and accumulation of stored beam if a setting can be found where injected bunches are captured in the storage ring's acceptance, while particles already stored in the machine are not ejected from the rings' acceptance. Fig. 6 shows the limits

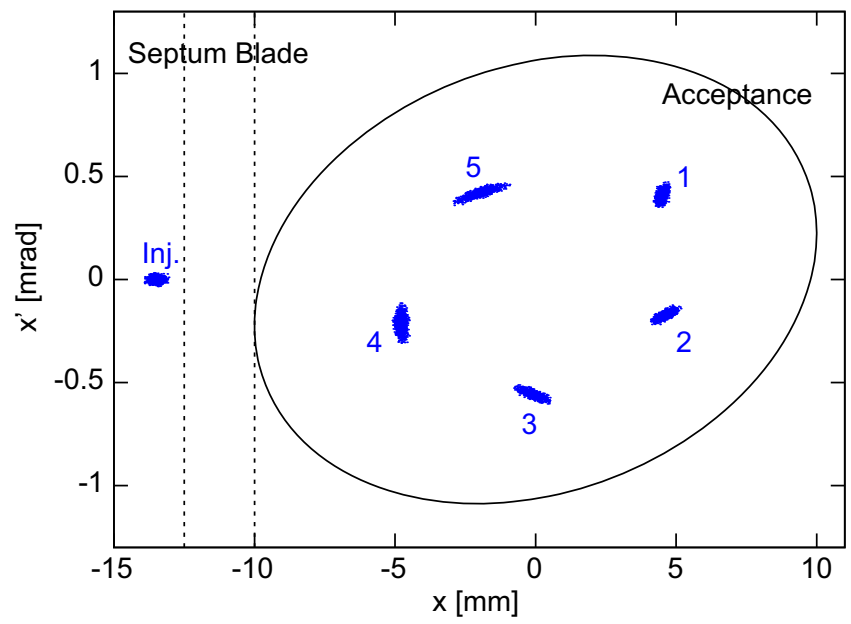

Fig. 4. DIMAD tracking data for injection, capture, and the first five turns in the storage ring at the IP. The dipole kicker has been set to $\theta_{\mathrm{ki}}=-3.9 \mathrm{mrad}$ in order to minimize the reduced invariant. The injected bunch is modeled with a Gaussian particle distribution using 1000 particles with $\varepsilon_{n}=10 \mathrm{~mm} \mathrm{mrad}, \sigma_{\delta}=0.1 \%$, and a cut-off at $3 \sigma$.
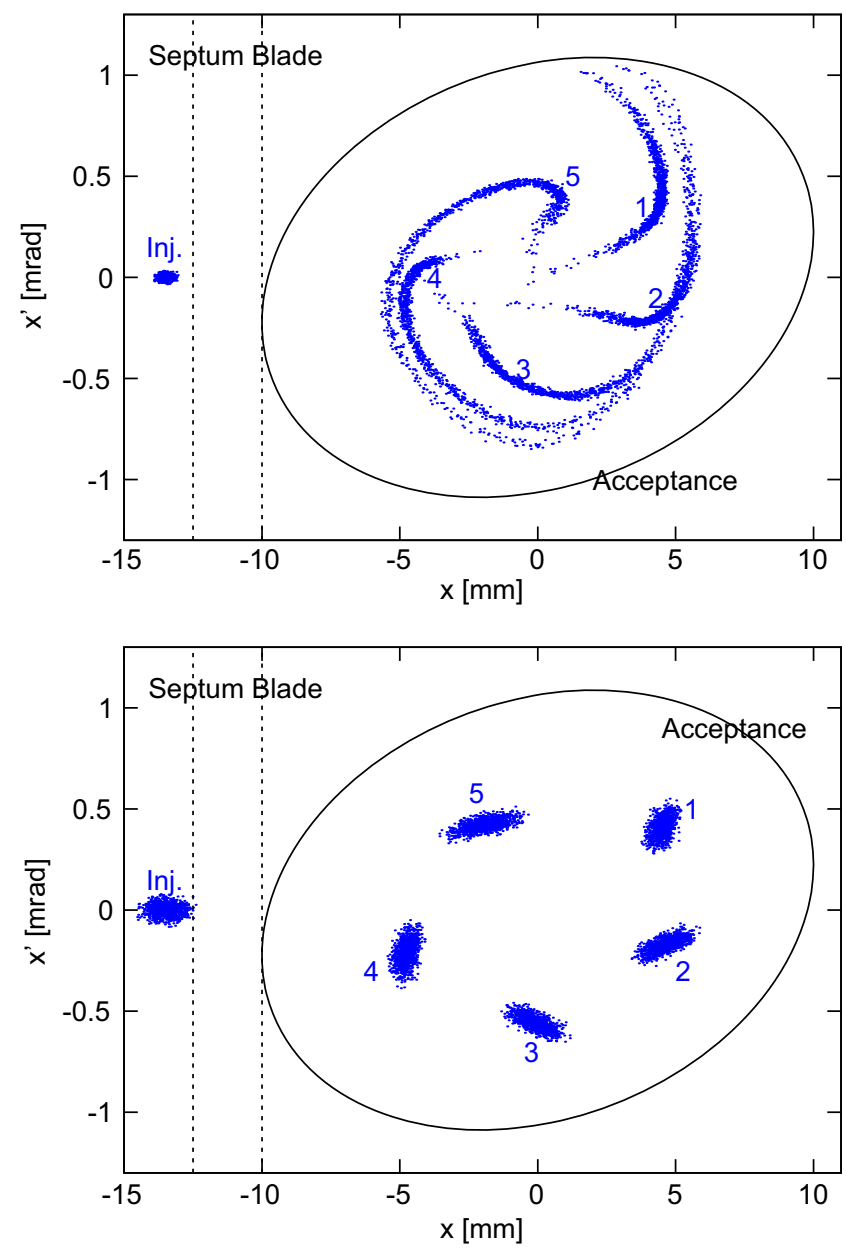

Fig. 5. DIMAD tracking data for mismatched injection. All parameters are identical to those used in Fig. 4 with the exception of an increased energy spread $\sigma_{\delta}=1.8 \%$ (top) and an emittance blow-up $\varepsilon_{n}=60 \mathrm{~mm}$ mrad (bottom).

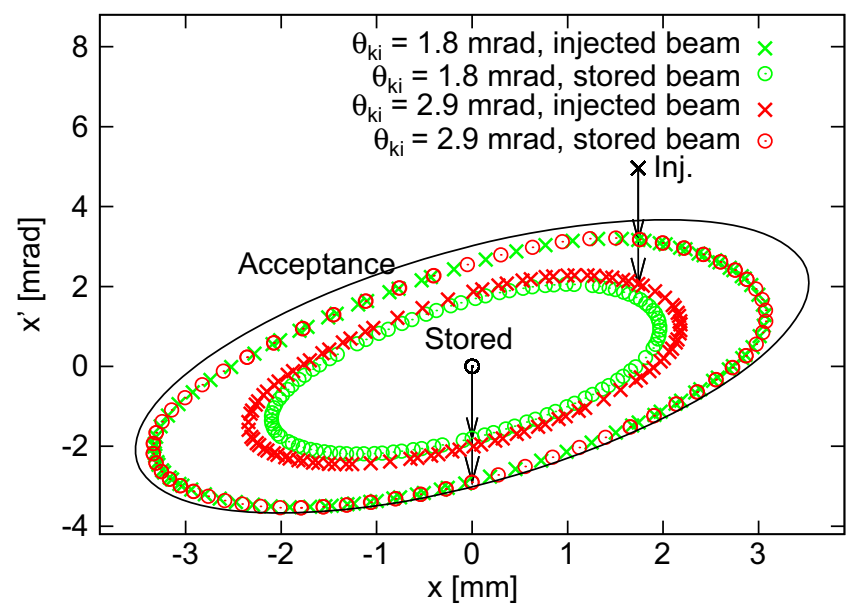

Fig. 6. Injection with the dipole kicker into the MAX IV $3 \mathrm{GeV}$ storage ring. The plot shows the minimum kick required to inject into the acceptance as well as the maximum kick that can be applied without ejecting stored beam particles out of the acceptance.

for such operation. The minimum kick required to capture injected particles is $\theta_{\mathrm{ki}}=-1.8 \mathrm{mrad}$. For such a kick stored beam particles are kicked to an invariant of $A_{\mathrm{inj}}^{2}=3.8 \mathrm{~mm}$ mrad which can easily be contained within the storage ring's acceptance. The maximum kick a stored beam particle can receive without being ejected form the acceptance is determined to be $\theta_{\mathrm{ki}}=-2.9 \mathrm{mrad}$. 


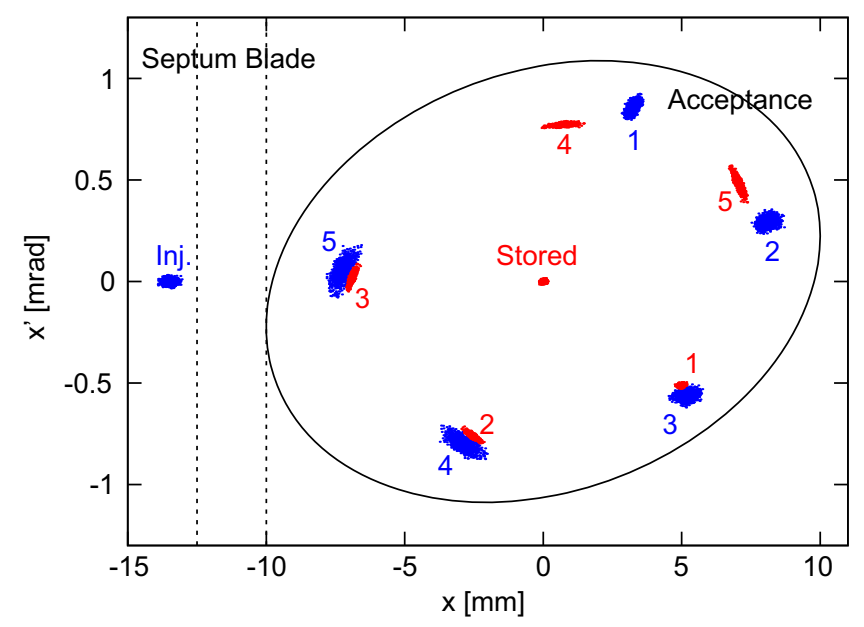

Fig. 7. DIMAD tracking demonstrates that the dipole kicker can capture injected particles without ejecting stored beam $\left(\theta_{\mathrm{ki}}=-2.3 \mathrm{mrad}\right)$ in the MAX IV $3 \mathrm{GeV}$ storage ring. The plot shows injection, capture, and the first five turns in the storage ring at the IP. Both the injected bunch and the stored beam are modeled with Gaussian particle distributions using 1000 particles and a cut-off at $3 \sigma$.

For such a kick injected particles are captured with a reduced invariant of $A_{\text {inj }}^{2}=3.8 \mathrm{~mm}$ mrad which can again easily be contained within the storage ring's acceptance. Therefore, it can be concluded that there is a roughly 1 mrad window for injection and accumulation with a single dipole kicker.

To demonstrate how such an injection actually succeeds in accumulating particles in the storage ring, a dipole kicker setting at the center of the above-mentioned window is chosen: $\theta_{\mathrm{ki}}=$ $-2.3 \mathrm{mrad}$, close to the optimum value for accumulation. The injection process together with the first five turns is modeled in DIMAD. The ensemble for the injected bunch used before is again applied, while the stored beam is modeled as a Gaussian particle distribution ( $n=1000$, cut-off at $3 \sigma$ ) using the bare lattice emittance of $\varepsilon_{x}=0.33 \mathrm{~nm}$ rad and lattice energy spread of $\sigma_{\delta}=0.77 \times$ $10^{-3}$. These assumptions are considered realistic because there are roughly seven damping times between consecutive injection shots. The results of such a tracking study are shown in Fig. 7.

While stored particles are kicked to considerable amplitudes, they can still be contained within the ring's acceptance. The stored beam size is blown up in the process; however, it has ample time to damp down (together with the injected beam oscillating at comparable amplitudes) until the next shot is injected. This demonstrates that while this injection method is not transparent and hence incompatible with user top-up operation, it is indeed a viable method to inject and accumulate beam in the storage ring. During commissioning and/or machine studies with limited stored current in decaying beam mode, having such a simple injection scheme using only a single dipole kicker is considered very robust and useful.

\subsection{On-axis injection}

For the reasons mentioned in the Introduction, on-axis injection is considered an important option in early commissioning. The first requirement for on-axis injection is to create a cross-over at the location of the dipole kicker. This can be achieved by giving the injected bunch a slightly negative angle at the IP. Because of the geometry of the septum's injection channel and the fact that the injected bunch passes through it with only $1 \mathrm{~mm}$ separation from the septum blade (the septum channel is approximately $1 \mathrm{~m}$ long), giving the injected bunch a negative angle at the IP also requires moving it away from the blade at the IP. Only in this way can a minimum clearance between the injected bunch and the septum blade at the entry of the septum's injection channel be

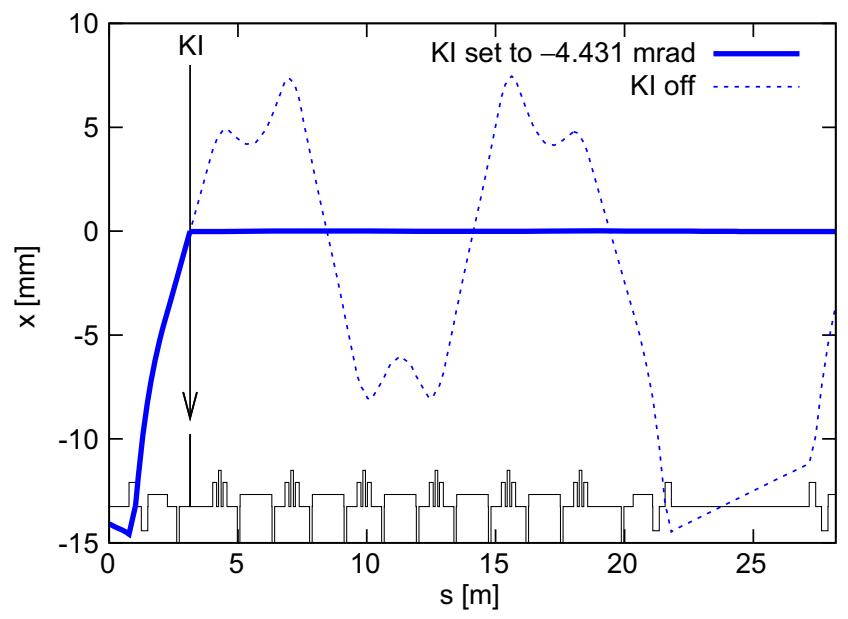

Fig. 8. On-axis injection with the dipole kicker into the MAX IV $3 \mathrm{GeV}$ storage ring. The bunch is injected at the IP with an angle of $x_{\text {IP }}^{\prime}=-0.59$ mrad. The plot shows the amplitude of the injected bunch from the IP through the first achromats. The reduction of amplitude with a dipole kick of $\theta_{\mathrm{ki}}=-4.4 \mathrm{mrad}$ is displayed.
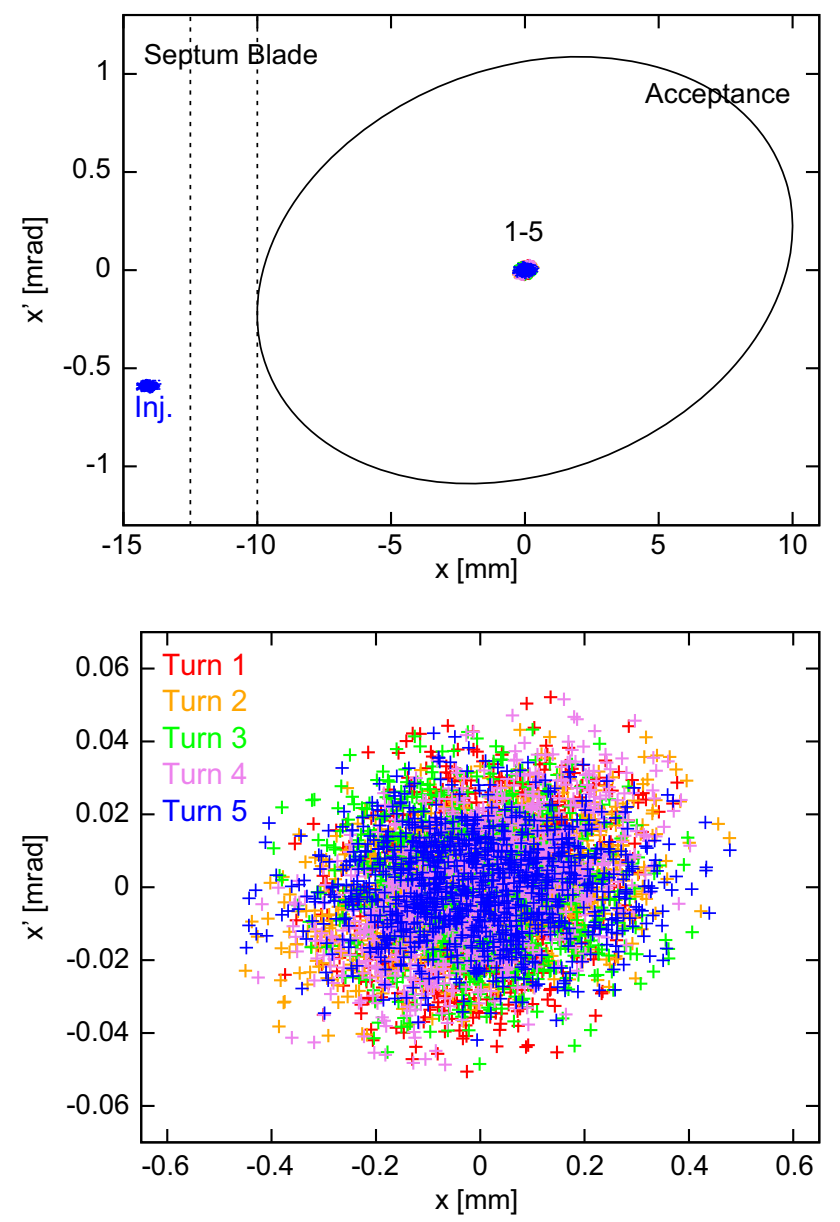

Fig. 9. DIMAD tracking data for on-axis injection, capture, and the first five turns in the storage ring at the IP. The dipole kicker has been set to $\theta_{\mathrm{ki}}=-4.4 \mathrm{mrad}$ in order to achieve zero reduced invariant. The injected bunch is modeled with a Gaussian particle distribution using 1000 particles with $\varepsilon_{n}=10 \mathrm{~mm}$ mrad, $\sigma_{\delta}=0.1 \%$, and a cut-off at $3 \sigma$. The first five turns are depicted with different colors. Top: the injection and capture process. Bottom: an enlarged view of the captured beam. (For interpretation of the references to color in this figure legend, the reader is referred to the web version of this article.)

ensured. Tracking studies show that a suitable set of parameters is $x_{\mathrm{inj}}=-14.09 \mathrm{~mm}$ and $x_{\mathrm{inj}}^{\prime}=-0.59 \mathrm{mrad}$ which generate $x_{\mathrm{ki}}=$ $0.00 \mathrm{~mm}$ and $x_{\mathrm{ki}}^{\prime}=4.43 \mathrm{mrad}$ at the dipole kicker without any 
expected losses in the septum's injection channel. If the dipole kicker is then set to $\theta_{\mathrm{ki}}=-4.43 \mathrm{mrad}$ the resulting reduced invariant becomes zero. This process is depicted in Fig. 8 where the negative angle at the IP can be clearly recognized.

A closer inspection using ensemble tracking at the IP reveals on-axis injection with this setting achieves zero reduced invariant while retaining the injected bunch's emittance. The results of such a study in DIMAD are shown in Fig. 9. Clearly on-axis injection can be used to inject into a severely misaligned ring or a ring with heavily mismatched optics (optics errors between the IP and the dipole kicker can however change the injection orbit which can then require retuning of the kicker) as could be encountered during early commissioning. In order to provide this option for commissioning, the transfer line will been equipped with sufficient BPMs and correctors in order to adjust the exact position and angle of the injected bunches at the IP.

Because of the zero reduced invariant, this method can also be applied in the case of an emittance or energy spread blow-up of the injected beam or an optics mismatch in the linac and transfer line. Again conditions that cannot entirely be ruled out during early injection.

\section{Injection into the $1.5 \mathrm{GeV}$ storage ring}

Analogous to the $3 \mathrm{GeV}$ storage ring, the IP in the $1.5 \mathrm{GeV}$ storage ring is defined as the magnetic end of the $10^{\circ}$ injection septum (1252 mm downstream of the center of the injection straight). Fig. 10 shows a schematic of the injection layout. The optics in the horizontal plane at this location are $\beta_{\text {inj }}=5.960 \mathrm{~m}$ and $\alpha_{\mathrm{inj}}=-0.220$. The lateral placement of the septum was again chosen so that the blade becomes the limiting horizontal aperture of the storage ring $A_{x}^{2}=32.06 \mathrm{~mm}$ mrad.

The injected bunch at the IP is at $x_{\mathrm{inj}}=-17 \mathrm{~mm}$ which gives an injected invariant of $A_{\text {inj }}^{2}=50.84 \mathrm{~mm}$ mrad. Fig. 11 (top) shows the amplitude of the injected bunch from the IP through the first two achromats and the dipole kicker in the third long straight. Ideally the dipole kicker would be installed closer to the IP, but since the injected amplitude in the second straight is too large (and
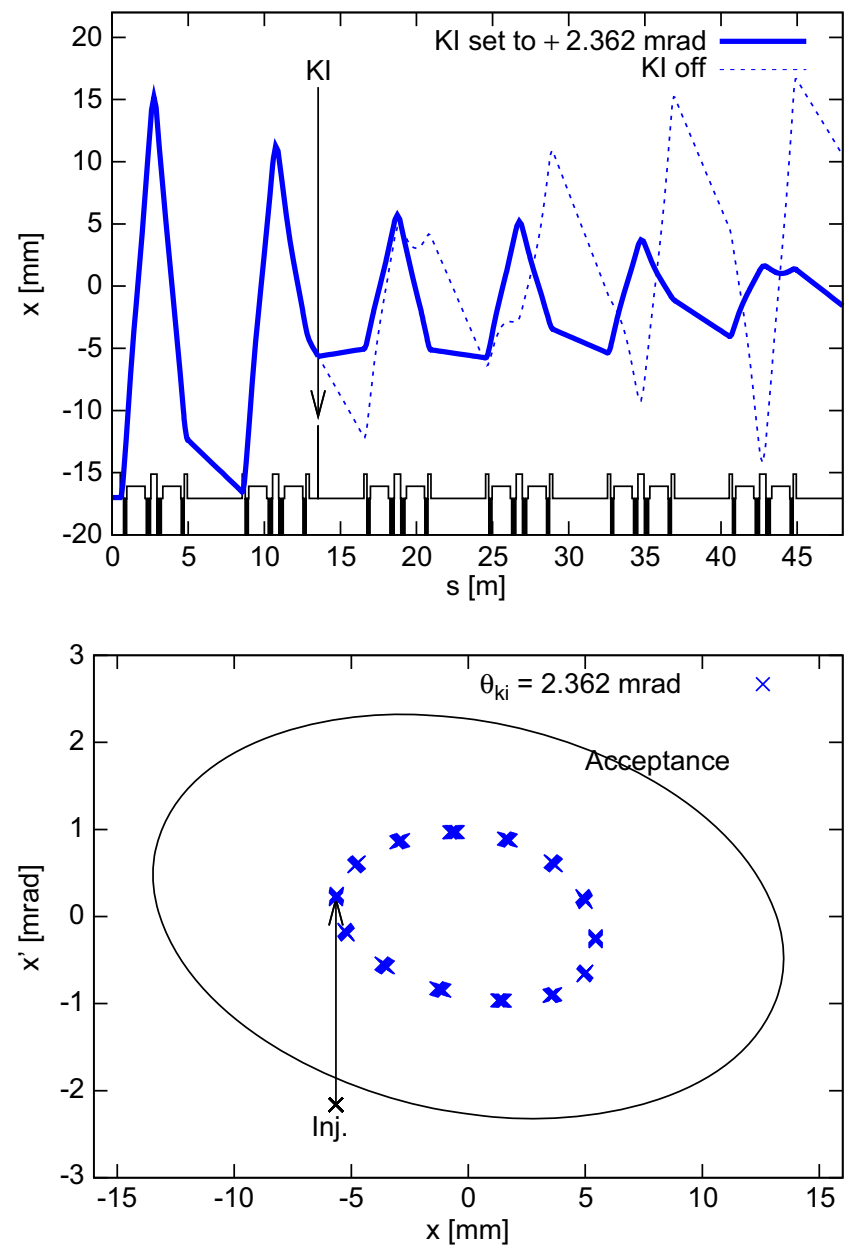

Fig. 11. Injection with the dipole kicker into the MAX IV $1.5 \mathrm{GeV}$ storage ring. Top: amplitude of the injected bunch from the IP through the first achromats. Bottom: capture and the first 100 turns in the storage ring are shown at the location of the dipole kicker. As demonstrated by the plot, setting the dipole kicker to $\theta_{\mathrm{ki}}=+2.4 \mathrm{mrad}$ minimizes the reduced invariant.
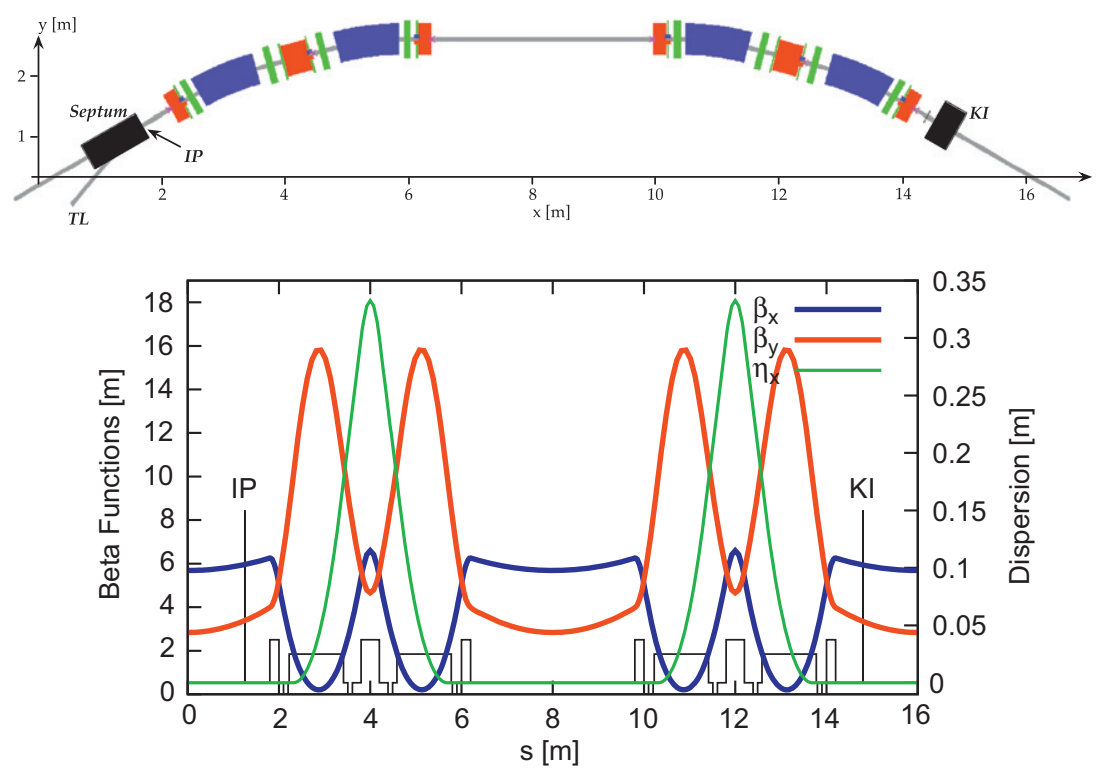

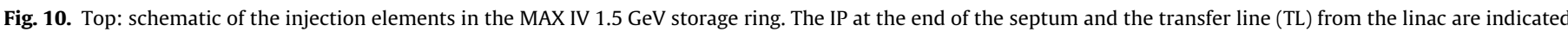

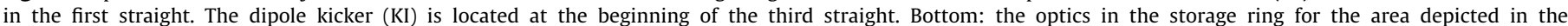
schematic above. 


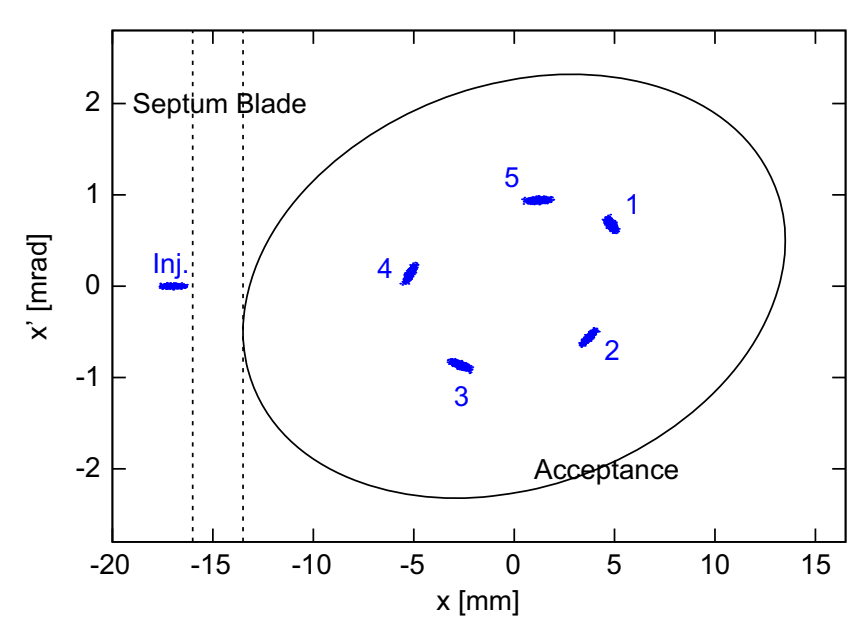

Fig. 12. DIMAD tracking data for injection, capture, and the first five turns in the storage ring at the IP. The dipole kicker has been set to $\theta_{\mathrm{ki}}=+2.4 \mathrm{mrad}$ in order to minimize the reduced invariant. The injected bunch is modeled with a Gaussian particle distribution using 1000 particles with $\varepsilon_{n}=10 \mathrm{~mm}$ mrad, $\sigma_{\delta}=0.1 \%$, and a cut-off at $3 \sigma$.

cannot be reduced sufficiently by applying moderate angles to the injected bunch at the IP), the third straight becomes an obvious candidate. The dipole injection kicker and the pulsed sextupole magnet [8] both have similar demands and hence end up competing for the same space in the third straight. It was therefore decided to install only one of these devices at a time. The dipole kicker will be installed first and used for early commissioning. Once the ring has been characterized and design optics and orbits have been achieved, the dipole kicker is replaced with the pulsed sextupole magnet so that the latter can be commissioned before using it for top-up injection during user operation.

The exact position of the center of the dipole kicker $(\mathrm{KI})$ is in the third straight, $1202 \mathrm{~mm}$ upstream of the center of the straight. At the location of KI the storage ring optics in the horizontal plane are $\beta_{\mathrm{ki}}=5.939 \mathrm{~m}$ and $\alpha_{\mathrm{ki}}=0.212$ and the coordinates of the injected bunch are $x_{\mathrm{ki}}=-5.654 \mathrm{~mm}$ and $x_{\mathrm{ki}}^{\prime}=-2.162 \mathrm{mrad}$. Tracking reveals that increasing the divergence of the injected bunch at KI by $x_{\mathrm{ki}}^{\prime}=-2.162 \rightarrow+0.20 \mathrm{mrad}$, i.e. $\theta_{\mathrm{ki}}=2.362 \mathrm{mrad}$, minimizes the resulting reduced invariant at $A_{\text {red }}^{2}=5.38 \mathrm{~mm}$ mrad. The phase space plot in Fig. 11 (bottom) demonstrates clearly why the minimum invariant is reached with this kick.

It should be noted that the reduced invariant achieved in this way is not only well within the storage ring acceptance $A_{x}$, it is also identical to what can be achieved with the pulsed sextupole magnet injection scheme [8]. In order to assess injection efficiency when using the dipole kicker in this manner, an ensemble of particles has been tracked from the IP and through the storage ring lattice for its first five turns. The particle ensemble (a Gaussian particle distribution, $n=1000$, cut-off at $3 \sigma$ ) is modeled using the expected parameters after acceleration from the thermionic rf-gun in the linac and transport through the transfer line. The normalized emittance of the injected bunch is assumed to be $\varepsilon_{n}=10 \mathrm{~mm}$ mrad (corresponding to $\varepsilon_{x}=3.4 \mathrm{~nm}$ rad), the energy spread is $\sigma_{\delta}=0.1 \%$. The transfer line optics are adjusted to deliver $\beta_{x}=21.133 \mathrm{~m}$ and $\alpha_{x}=-0.002$ at the IP [12]. The results of such a tracking study using DIMAD are shown in Fig. 12. Injection and capture in the storage ring are shown for the above detailed dipole kicker setting. The plot shows the data at the IP. The position of the septum is indicated. In this process no particles are lost which indicates injection efficiency should be very high.

\subsection{Tolerances}

During actual operation, the linac could have slightly different optics or the extracted bunch could suffer an emittance and/or
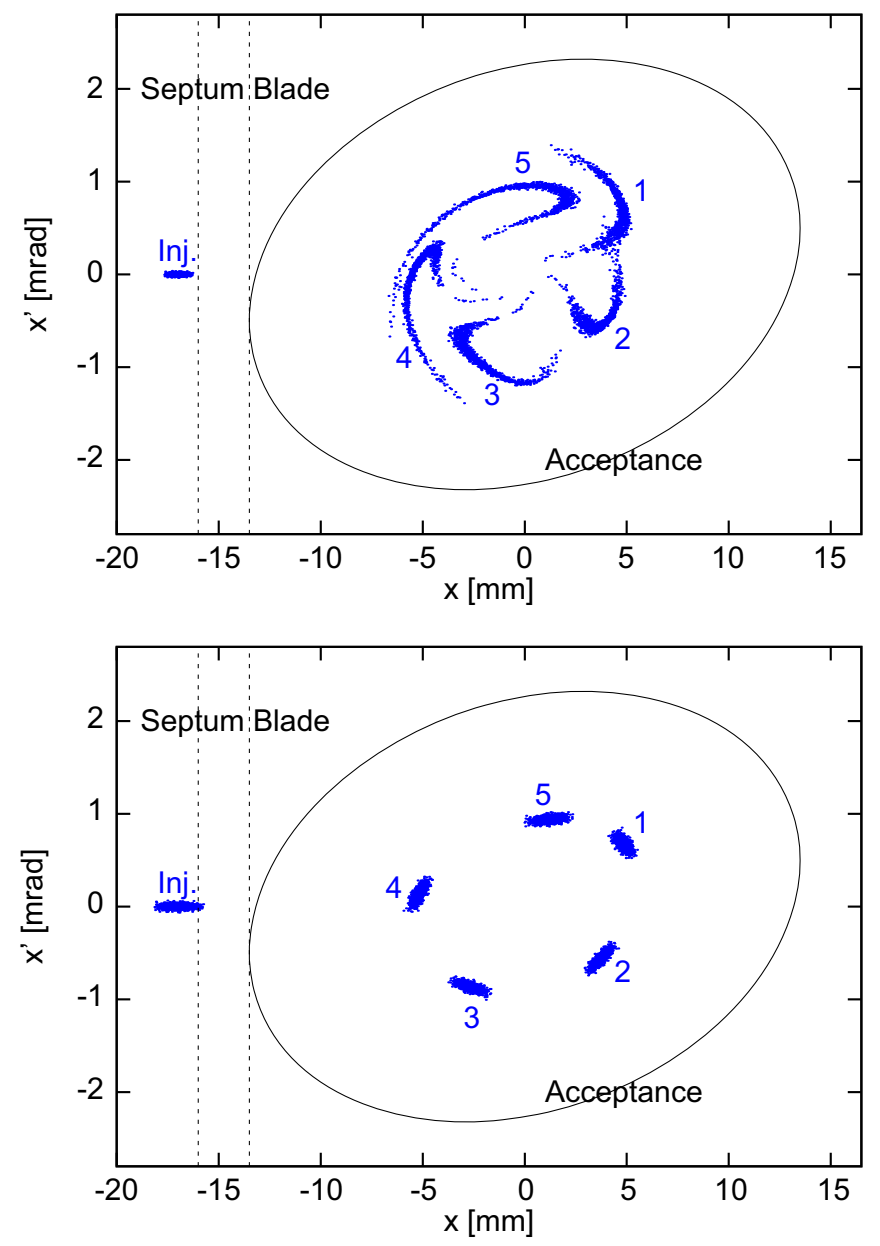

Fig. 13. DIMAD tracking data for mismatched injection. All parameters are identical to those used in Fig. 12 with the exception of an increased energy spread $\sigma_{\delta}=2.0 \%$ (top) and an emittance blow-up $\varepsilon_{n}=30 \mathrm{~mm}$ mrad (bottom).

energy spread increase. In order to assess tolerances for linac operation, two extreme cases are displayed in Fig. 13 where capture efficiency is no longer $100 \%$. A situation where the energy spread of the injected bunch has increased to $\sigma_{\delta}=2.0 \%$ (which is actually well beyond the energy acceptance of the transfer line) shows smearing out of the injected bunch, but the tails have not started to scrape the acceptance. The case with a three-fold increased emittance of $\varepsilon_{n}=30 \mathrm{~mm}$ mrad simulates emittance blow-up in the linac as well as optics mismatches up to the IP. In this situation a blow-up of the bunch is observed, without significant distortion however. Any further increase of emittance will lead to particle loss on the septum blade.

\subsection{Accumulation}

As already detailed in the Introduction, the dipole kicker can be used for injection and accumulation of stored beam if a setting can be found where injected bunches are capture in the storage ring's acceptance, while particles already stored in the machine are not ejected from the rings' acceptance. Fig. 14 shows the limits for such operation. The minimum kick required to capture injected particles is $\theta_{\mathrm{ki}}=+0.8 \mathrm{mrad}$. For such a kick stored beam particles are kicked to an invariant of $A_{\mathrm{inj}}^{2}=3.80 \mathrm{~mm}$ mrad which can easily be contained within the storage ring's acceptance. The maximum kick a stored beam particle can receive without being ejected form the acceptance is determined to be $\theta_{\mathrm{ki}}=+2.1 \mathrm{mrad}$. For such a kick injected particles are captured with a reduced 


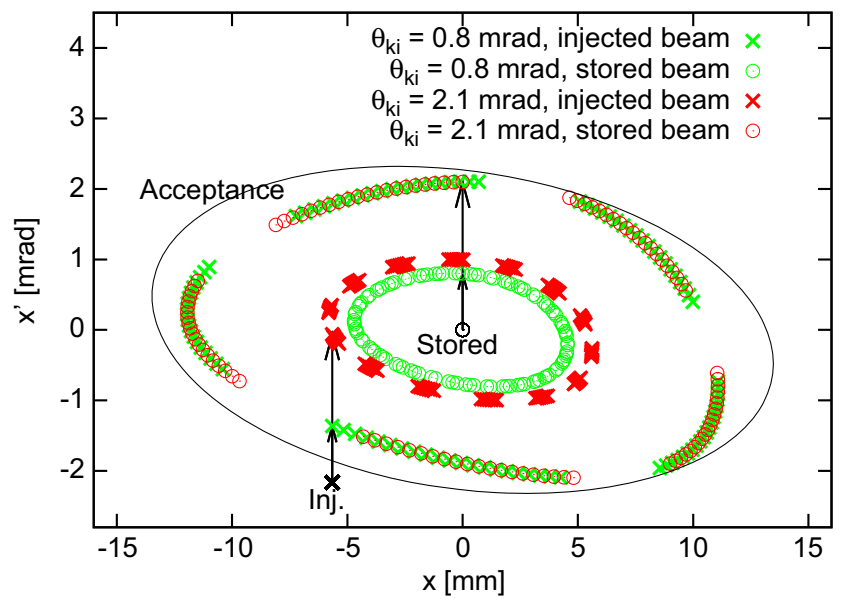

Fig. 14. Injection with the dipole kicker into the MAX IV $1.5 \mathrm{GeV}$ storage ring. The plot shows the minimum kick required to inject into the acceptance as well as the maximum kick that can be applied without ejecting stored beam particles out of the acceptance.

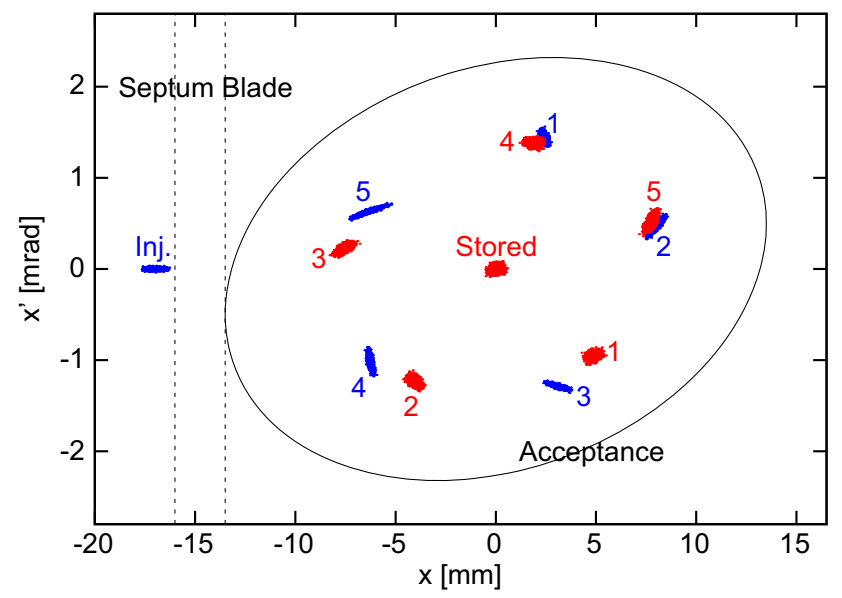

Fig. 15. DIMAD tracking demonstrates that the dipole kicker can capture injected particles without ejecting stored beam $\left(\theta_{\mathrm{ki}}=+1.4 \mathrm{mrad}\right)$ in the MAX IV $1.5 \mathrm{GeV}$ storage ring. The plot shows injection, capture, and the first five turns in the storage ring at the IP. Both the injected bunch and the stored beam are modeled with Gaussian particle distributions using 1000 particles and a cut-off at $3 \sigma$.

invariant of $A_{\text {inj }}^{2}=5.79 \mathrm{~mm}$ mrad which can again easily be contained within the storage ring's acceptance. Therefore, it can be concluded that there is a roughly $1.3 \mathrm{mrad}$ window for injection and accumulation with a single dipole kicker.

To demonstrate how such an injection actually succeeds in accumulating particles in the storage ring, a dipole kicker setting at the center of the above-mentioned window is chosen: $\theta_{\mathrm{ki}}=+1.4 \mathrm{mrad}$. The injection process together with the first five turns is modeled in DIMAD. The ensemble for the injected bunch used before is again applied, while the stored beam is modeled as a Gaussian particle distribution ( $n=1000$, cut-off at $3 \sigma$ ) using the bare lattice emittance of $\varepsilon_{x}=6 \mathrm{~nm}$ rad and lattice energy spread of $\sigma_{\delta}=0.75 \times 10^{-3}$. These assumptions are considered realistic because there are roughly 14 damping times between consecutive injection shots. The results of such a tracking study are shown in Fig. 15.

While stored particles are kicked to several-mm amplitudes, they can still be contained within the ring's acceptance. The stored beam size is hardly blown up in the process and has ample time to damp down (together with the injected beam oscillating at comparable amplitudes) until the next shot is injected. This demonstrates that while this injection method is not transparent and hence incompatible with user top-up operation, it is indeed a viable method to inject and accumulate beam in the storage ring. During commissioning and/or machine studies with limited stored current in decaying beam mode, having such a simple injection scheme using only a single dipole kicker is considered very robust and useful.

\subsection{Solaris considerations}

The MAX IV $1.5 \mathrm{GeV}$ storage ring will be built twice. Besides serving UV and IR users at MAX IV, a copy of the ring will be built in Krakow, Poland, at the heart of the new Solaris facility [13]. The $1.5 \mathrm{GeV}$ Solaris storage ring will resemble the $1.5 \mathrm{GeV}$ MAX IV ring as closely as possible; however, it will not have a full-energy injector. Instead, the Solaris linac will inject particles into the storage ring at $550 \mathrm{MeV}$ where accumulation occurs [14]. Once the desired current has been reached in the storage ring, the energy is ramped to $1.5 \mathrm{GeV}$. Therefore, regular Solaris user operation foresees decaying beam mode for which the single dipole kicker injection scheme is perfectly adequate (under the assumption that coherent oscillations of the stored beam during injection do not give rise to significant instabilities at large current). The tracking studies performed above can be repeated for the Solaris injection energy of $550 \mathrm{MeV}$ as well as the Solaris linac and transfer line optics $\left(\varepsilon_{n}=\right.$ $10 \mathrm{~mm}$ mrad, $\left.\sigma_{\delta}=0.1 \%, \beta_{x, \text { inj }}=5.517 \mathrm{~m}, \alpha_{x, \text { inj }}=-0.137\right)$. Fig. 16 shows results of such a study. Compared to the case at $1.5 \mathrm{GeV}$ (cf. Fig. 15), the beam size of the injected bunch is larger because of the increased emittance $\left(\varepsilon_{x}=9.3 \mathrm{~nm}\right.$ rad) but the stored beam emittance and hence beam size is smaller $\left(\varepsilon_{x}=0.8 \mathrm{~nm}\right.$ rad at $550 \mathrm{MeV}$ ). Otherwise the injection process is very similar and capture efficiency will again be very high. Slightly moving the injected beam away from the septum blade could be contemplated to ensure no particles are lost in the septum's injection channel.

A significant difference for Solaris is the damping time at $550 \mathrm{MeV}$ which is on the order of $150 \mathrm{~ms}$. If the MAX IV injection repetition rate of $10 \mathrm{~Hz}$ were used, the injected and stored beams would not be given enough time to damp down to zero reduced amplitude, bare lattice emittance, and natural energy spread $\left(\sigma_{\delta}=0.27 \times 10^{-3}\right.$ at $\left.550 \mathrm{MeV}\right)$. When the next shot is injected and the dipole kicker is triggered, the previously accumulated bunches at their non-zero invariants could be ejected from the acceptance. This problem can be solved by reducing the repetition rate to roughly $2-3 \mathrm{~Hz}$ so that there remain two to three damping times between injections. Despite such a reduction of injection

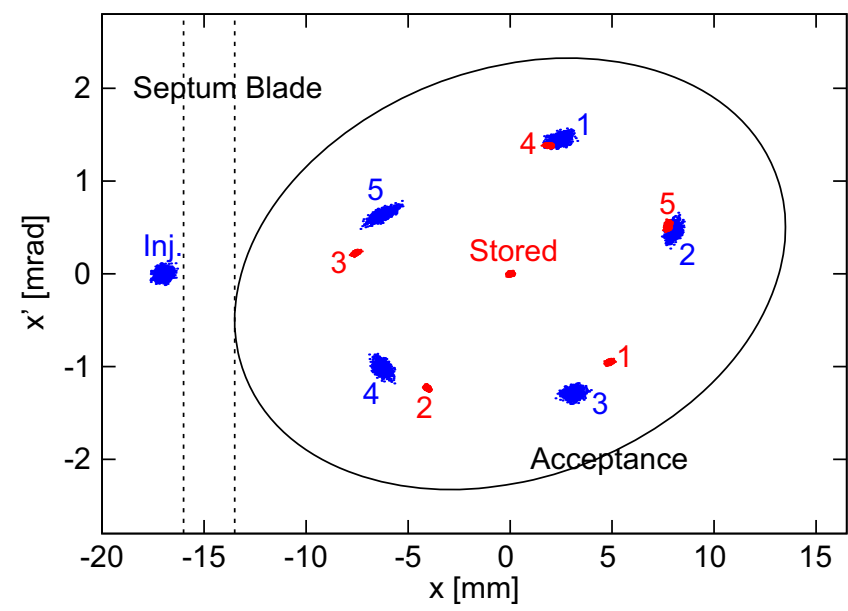

Fig. 16. DIMAD tracking for the Solaris injection energy of $550 \mathrm{MeV}$ demonstrates that the dipole kicker can capture injected particles without ejecting stored beam $\left(\theta_{\mathrm{ki}}=+1.4 \mathrm{mrad}\right)$. The plot shows injection, capture, and the first five turns in the storage ring at the IP. Both the injected bunch and the stored beam are modeled with Gaussian particle distributions using 1000 particles and a cut-off at $3 \sigma$. 
repetition rate, assuming $80 \%$ overall injection efficiency, the Solaris ring can still be filled to $500 \mathrm{~mA}$ stored current in roughly 5 min.

\subsection{On-axis injection}

As for the $3 \mathrm{GeV}$ storage ring, on-axis injection is considered an important option in early commissioning of the $1.5 \mathrm{GeV}$ storage ring. The first requirement for on-axis injection is to create a crossover at the location of the dipole kicker. This can be achieved by giving the injected bunch a slightly negative angle at the IP. As in the case of the $3 \mathrm{GeV}$ ring, because of the geometry of the septum's injection channel, giving the injected bunch a negative angle at the IP also requires moving it away from the blade at the IP. Tracking studies show that a suitable set of parameters is $x_{\text {inj }}=-17.84 \mathrm{~mm}$ and $x_{\mathrm{inj}}^{\prime}=-0.84 \mathrm{mrad}$ which generate $x_{\mathrm{ki}}=0.00 \mathrm{~mm}$ and $x_{\mathrm{ki}}^{\prime}=$ $-2.85 \mathrm{mrad}$ at the dipole kicker without any expected losses in the septum's injection channel. If the dipole kicker is then set to $\theta_{\mathrm{ki}}=+2.85 \mathrm{mrad}$ the resulting reduced invariant becomes zero. This process is depicted in Fig. 17 where the negative angle at the IP can be clearly recognized.

A closer inspection using ensemble tracking at the IP reveals on-axis injection with this setting achieves zero reduced invariant while almost retaining the injected bunch's emittance. The results of such a study in DIMAD are shown in Fig. 18. Clearly on-axis injection can be used to inject into a severely misaligned ring or a ring with heavily mismatched optics (optics errors between the IP and the dipole kicker can however change the injection orbit which can then require retuning of the kicker) as could be encountered during early commissioning. In order to provide this option for commissioning, the transfer line will been equipped with sufficient BPMs and correctors in order to adjust the exact position and angle of the injected bunches at the IP.

Because of the zero reduced invariant, this method can also be applied in the case of an emittance or energy spread blow-up of the injected beam or an optics mismatch in the linac and transfer line. Again conditions that cannot entirely be ruled out during early injection.

\subsection{Two-turn injection}

Because of the short revolution period of the $1.5 \mathrm{GeV}$ storage ring, the required dipole kicker pulse length becomes quite demanding. It

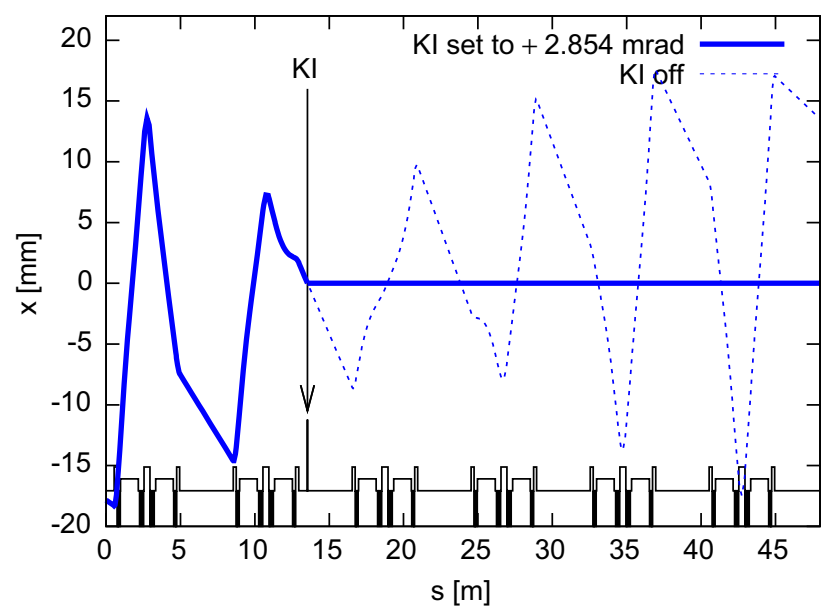

Fig. 17. On-axis injection with the dipole kicker into the MAX IV $1.5 \mathrm{GeV}$ storage ring. The bunch is injected at the IP with an angle of $x_{\mathrm{IP}}^{\prime}=-0.84 \mathrm{mrad}$. The plot shows the amplitude of the injected bunch from the IP through the first achromats. The reduction of amplitude with a dipole kick of $\theta_{\mathrm{ki}}=+2.9 \mathrm{mrad}$ is displayed.
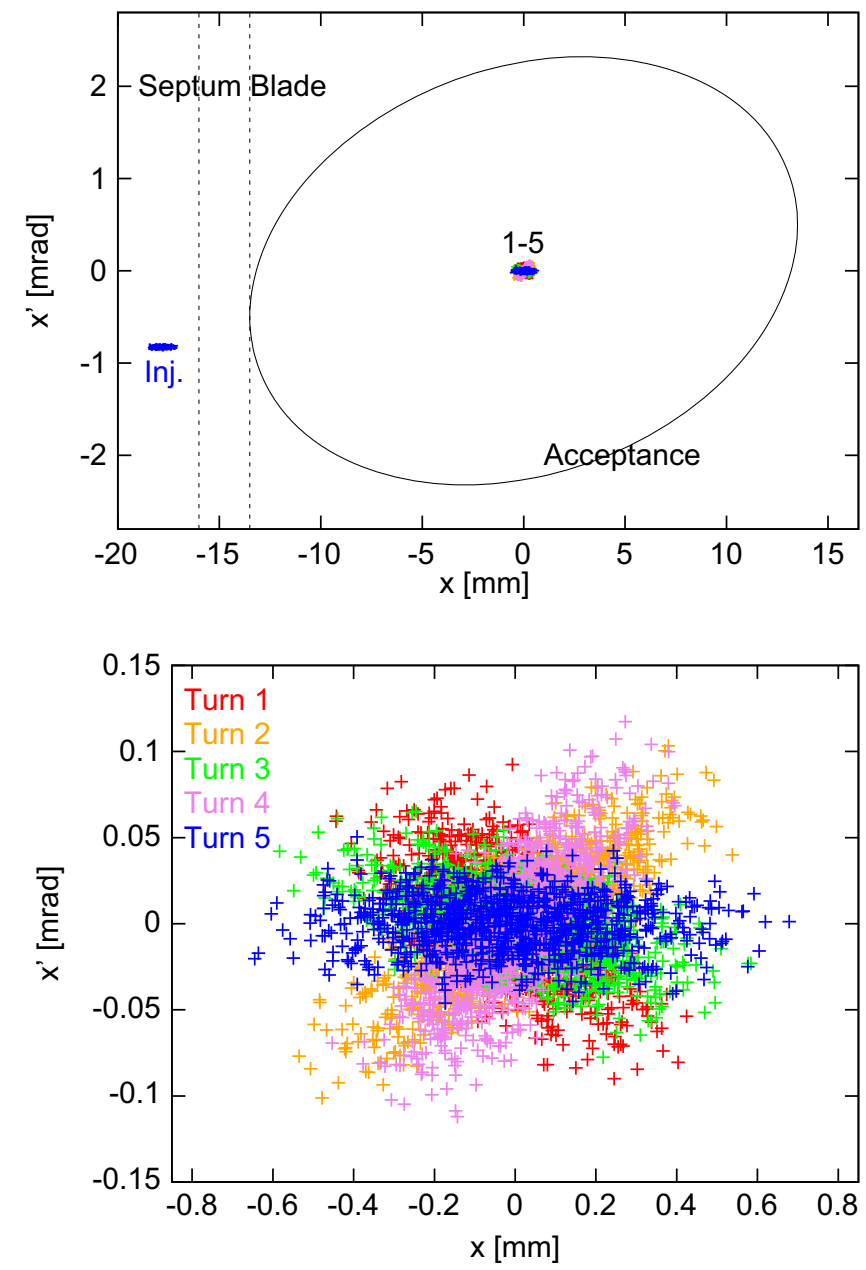

Fig. 18. DIMAD tracking data for on-axis injection, capture, and the first five turns in the storage ring at the IP. The dipole kicker has been set to $\theta_{\mathrm{ki}}=+2.85 \mathrm{mrad}$ in order to achieve zero reduced invariant. The injected bunch is modeled with a Gaussian particle distribution using 1000 particles with $\varepsilon_{n}=10 \mathrm{~mm}$ mrad, $\sigma_{\delta}=0.1 \%$, and a cut-off at $3 \sigma$. The first five turns are depicted with different colors. Top: the injection and capture process. Bottom: an enlarged view of the captured beam. (For interpretation of the references to color in this figure legend, the reader is referred to the web version of this article.)

is therefore of interest to investigate if injection can be performed over two turns thus relaxing the pulse length requirement from $640 \mathrm{~ns}$ to $1.28 \mu \mathrm{s}$. In such a scheme the dipole kicker pulse, which will be assumed to be a half-sine here, extends over four instead of two revolution periods. When the injected bunch passes the dipole kicker the first time it receives the first kick with strength $\theta_{\mathrm{ki}}^{(0)}$. On the next turn, when the injected bunch passes the dipole kicker it receives a second, reduced kick $\theta_{\mathrm{ki}}^{(1)}=\sin (3 \pi / 4) \times \theta_{\mathrm{ki}}^{(0)}$. The challenge is to provide sufficient kick during the first passage to kick the injected bunch within the storage ring acceptance, without supplying so much kick strength that the second kick (which has the same sign) kicks this just captured bunch out of the acceptance again.

For the $1.5 \mathrm{GeV}$ storage ring tracking studies have revealed that the kick strength that delivers the minimum reduced invariant after both kicks is $\theta_{\mathrm{ki}}^{(0)}=+2.0 \mathrm{mrad}$. Unfortunately the resulting reduced invariant is roughly $A_{\mathrm{red}}^{2}=33 \mathrm{~mm}$ mrad which is just above the storage ring acceptance. Although the injected bunch can be captured during the first turn, it is likely to be lost on subsequent turns. Furthermore, any beam already stored in the machine is ejected from the storage ring acceptance during the second kick. One way to get around this problem would be to change the working point during injection. Although possible, it is 
not deemed practical. The pulse duration requirement will therefore remain below two revolution periods (cf. Section 5.2).

\section{Technical design issues}

\subsection{Magnet and vacuum chamber design}

The dipole kicker magnet specifications are derived from the requirements presented above. The length of the dipole kicker has to be determined taking into consideration available space in the storage ring, pole-tip field and stored energy. Because of the short pulse duration, especially in the $1.5 \mathrm{GeV}$ storage ring, the inductance of the dipole kicker can become rather large; hence a reduction of the amount of stored energy in the magnet is crucial to prevent pulser voltages from becoming too large. A magnetic length of $0.3 \mathrm{~m}$ for the dipole kickers in both MAX IV storage rings was considered a reasonable compromise. Based on this choice a summary of the magnet specifications is displayed in Table 1. Besides nominal values, it contains specifications for injection with accumulation.

Taking into account the design acceptance of the storage rings as well as the nominal vacuum chamber design [15], beam stayclear areas for the dipole kicker chambers can be derived. Given as full apertures $(\mathrm{H} \times \mathrm{V})$ these are $8 \times 16.5 \mathrm{~mm}[28 \times 10 \mathrm{~mm}]$ for the two rings. In order to prevent synchrotron radiation from an upstream dipole from hitting the chamber walls, the beam stayclear area is enlarged and the area is moved off-center with respect to the stored beam. This results in full apertures of $31 \times$ $16.5 \mathrm{~mm}[41 \times 15 \mathrm{~mm}]$ where the stored beam is located at $-4.5 \mathrm{~mm}$ ( $-5.5 \mathrm{~mm})$ from the chamber center [16]. For the $1.5 \mathrm{GeV}$ storage ring the vertical aperture has been increased in order to reduce the amount of tapering required from the nominal vacuum chamber. Taking into account these chamber dimensions as well as the position of the injected beam, a good-field region for the dipole kicker can be specified as $\pm 7 \mathrm{~mm}[ \pm 14 \mathrm{~mm}$ ].

In order to allow the quickly changing magnetic fields of the dipole kicker to penetrate the vacuum vessel without significant distortion from eddy currents, a ceramic tube is chosen as a vacuum chamber. This vessel has to be coated internally with a thin conducting layer so image charges do not sense a discontinuity in the chamber. Such a coating can however attenuate the applied magnetic field and distort the pulse shape. Preliminary calculations show that a $3 \mu \mathrm{m}(0.5 \mu \mathrm{m})$ Ti coating restricts heating (from eddy currents and image currents at $500 \mathrm{~mA}$ stored beam) of the chamber to levels around $99 \mathrm{~W} / \mathrm{m}(332 \mathrm{~W} / \mathrm{m})$ without deforming the pulse shape significantly or attenuating the field strength by more than $\approx 1 \%$ [17]. The thickness of a suitable ceramic vessel is estimated to be $6 \mathrm{~mm}(5 \mathrm{~mm})$ so that a minimum magnetic full aperture of $57 \times 32.5 \mathrm{~mm}(65 \times 29 \mathrm{~mm})$ is derived.

The storage ring vacuum chambers outside the injection straight and dipole kicker area do not need to be modified for injection with

Table 1

Dipole kicker magnet parameters for injection into the MAX IV and Solaris storage rings.

\begin{tabular}{llll}
\hline Mode of injection & $B(\mathrm{~T})$ & $L(\mathrm{~m})$ & $\tau(\mu \mathrm{s})$ \\
\hline $3 \mathrm{GeV}$ injection dipole, nominal & 0.131 & 0.3 & 3.5 \\
Accumulation & 0.077 & 0.3 & 3.5 \\
On-axis injection & 0.148 & 0.3 & 3.5 \\
$1.5 \mathrm{GeV}$ injection dipole, nominal & 0.039 & 0.3 & 0.64 \\
Accumulation & 0.023 & 0.3 & 0.64 \\
On-axis injection & 0.048 & 0.3 & 0.64 \\
Solaris injection & 0.009 & 0.3 & 0.64 \\
\hline
\end{tabular}

a single dipole kicker. Although the injected bunches in the $1.5 \mathrm{GeV}$ storage ring reach significant amplitudes between the IP and the dipole kicker (cf. Figs. 11 (top) and 17), these amplitudes are still within the acceptance set by physical aperture limitations of the standard vacuum chambers and absorbers.

\subsection{Pulser considerations}

With these properties of the dipole kicker magnet and its vacuum chamber, the pulser specifications can be set. Pulser requirements are summarized in Table 2. Pulse duration and shape are determined by the beam dynamics considerations mentioned in the previous sections. For single-turn injection the pulse shape is in principle not critical as long as fall time is short compared to the revolution period. It is however quite common to use half-sine pulses to drive dipole kickers. The base length of such a half-sine can be set to as much as two revolution periods. The pulse maximum is adjusted to coincide with arrival of the injected bunch at the dipole kicker. While such a pulse should be perfectly suitable for injection of bunches in to the MAX IV storage rings, it does have interesting implications for beam accumulation, since such a pulse will kick bunches in the stored beam twice and with different amplitudes depending on the bucket number. The effect of such a pulse shape on beam accumulation with a single dipole kicker shall be described in Appendix A.

While arrival time of the injected bunches with respect to the storage ring rf is critical [8], combined timing jitter of the dipole kicker pulse with respect to the injected bunch is more relaxed with better than \pm 5 ns over extended periods of time (typically 1 week). A repetition rate of $10 \mathrm{~Hz}$ is derived from the damping time in the storage rings. This results in 5-10 damping times between injection shots in the $3 \mathrm{GeV}$ storage ring (depending on the installed insertion devices and gap settings) and more than 10 damping times in the $1.5 \mathrm{GeV}$ storage ring. A reduced injection repetition rate can be applied for Solaris' $550 \mathrm{MeV}$ injection energy as discussed in Section 4.3.

Finally, it should be noted that possible pulser malfunction has no direct implications for machine protection or safety in the MAX IV storage rings. Injected bunches that do not receive the proper kick from the pulsed dipole will be lost on the vacuum chamber within the first turn. Because of the installation of a permanent magnet in each beamline front end [18], even grossly mis-steered bunches cannot be injected down a beamline regardless of shutter positions.

\subsection{Horizontal pinger operation}

The dipole injection kickers can also be used as an economical alternative to dedicated pinger magnets for the horizontal plane. In this way only pinger magnets for the vertical plane need to be procured. Since it is expected that pinger operation is applied only during dedicated shifts for machine studies, the storage rings can be operated in a single-bunch mode (the linac injector to the MAX

Table 2

Pulser requirements for dipole injection into the MAX IV $3 \mathrm{GeV}(1.5 \mathrm{GeV})$ storage ring.

\begin{tabular}{ll}
\hline Pulse length & $<3.5 \mu \mathrm{s}(640 \mathrm{~ns})$ \\
Fall time & $<1.8 \mu \mathrm{s}(320 \mathrm{~ns})$ \\
Pulse-to-pulse ampl. fluctuations within & $\pm 0.1 \%$ \\
Long-term amplitude within & $\pm 1 \%$ \\
Undershoot duration & $<1.5 \mu \mathrm{s}(0.2 \mu \mathrm{s})$ \\
Undershoot amplitude (w.r.t. main pulse) & $<10 \%$ \\
Timing jitter within & $\pm 5 \mathrm{~ns}$ \\
Maximum repetition rate & $10 \mathrm{~Hz}$ \\
\hline
\end{tabular}


IV storage rings can be set to inject into a single storage ring bucket) for pinger experiments. The stored beam then consists of only a single bunch that receives a single kick of well-defined amplitude from the dipole kicker during one passage. This kick excites coherent betatron oscillations of the stored bunch in the machine which can then be analyzed using the MAX IV BPM system operated in turn-by-turn mode [7].

Compared to nominal injection kick settings, some extra dipole kicker strength can be allocated so the pinger can excite even off-centered and/or mismatched beams to the limits of the storage ring acceptance. For the $3 \mathrm{GeV}$ storage ring $3.3 \mathrm{mrad}$ are required for such horizontal pinger operation. This is well bellow the $4.4 \mathrm{mrad}$ already required for on-axis injection. In the $1.5 \mathrm{GeV}$ ring the dipole injection kicker is moved to the injection straight for use as horizontal pinger magnet once the pulsed sextupole magnet is installed. Horizontal pinger operation calls for $2.3 \mathrm{mrad}$ which is what is already required for nominal injection to minimum reduced invariant and well below what is required for on-axis injection. Thus, the conclusion is that with a kick specification of $4.5 \mathrm{mrad}$ ( $2.9 \mathrm{mrad})$ for the $3 \mathrm{GeV}(1.5 \mathrm{GeV})$ dipole kicker, all discussed modes of operation will become available.

\section{Acknowledgments}

The author would like to thank Mikael Eriksson (MAX-lab), Les Dallin (CLS), and Peter Kuske (HZB) for many stimulating exchanges on alternative injection ideas as well as their support and encouragement. The author is grateful to Les Dallin for proofreading of the manuscript.

\section{Appendix A. Effect of a half-sine pulse on beam accumulation}

A simple approach to single-turn injection using the longest possible pulse, consists of using a half-sine pulse with a base length corresponding to two revolution periods. This is depicted schematically in Fig. A1. If the pulse maximum is adjusted to coincide with arrival of the injected bunch at the dipole kicker, the injected bunch is kicked exactly once and by the design kick strength. Particles in the stored beam are also only kicked once (and by the same amplitude) if they are in the same storage ring bucket as the injected bunch is being captured in. However, stored beam in all other buckets will be kicked twice and by

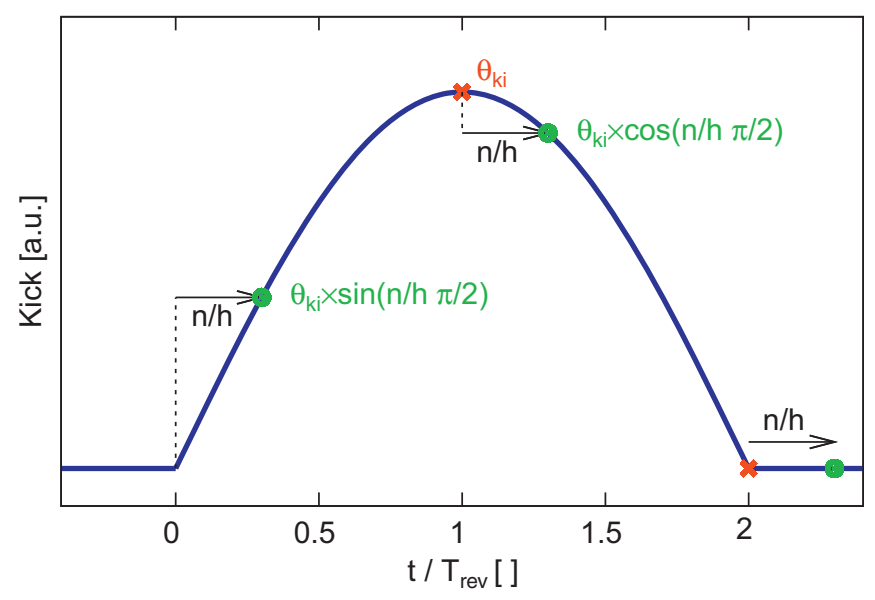

Fig. A1. Schematic depicting a half-sine kicker pulse and kicks applied to the injected beam (here in bucket 0 ; crosses) and stored beam particles in bucket $n$ (circles). $h$ is the harmonic number. two different amplitudes (except for bucket $n=h / 2$ where the kick amplitude is $\theta_{\mathrm{ki}} / \sqrt{2}$ for both kicks).

It can be shown that kicking stored beam particles twice leads to an increase of the stored beam invariant. Following the notation introduced in Section 2, the invariant of the stored beam after both kicks is given by

$A_{\mathrm{st}}^{2}=\beta_{\mathrm{ki}} \theta_{\mathrm{ki}}^{2}\left(1+\cos 2 \pi v_{x} \sin 2 \phi\right)$

where $v_{x}$ is the horizontal tune and $\phi=(n / h)(\pi / 2)$ the phase advance of the stored beam in bucket $n$ with respect to the injected beam, assuming injection is taking place into bucket 0 (cf. Fig. A1). The first term in Eq. (A.1) describes the kick to the stored beam $\beta_{\mathrm{ki}} \theta_{\mathrm{ki}}^{2}$ if it is in the same bucket as the one into which beam is being injected. The second term in Eq. (A.1) describes the growth of the invariant as a consequence of receiving two kicks. The relative growth of the invariant is then simply expressed as

$\Delta=\cos 2 \pi v_{x} \sin 2 \phi$.

From Eq. (A.2) it becomes obvious that this effect is least detrimental for a quarter-integer fractional horizontal tune. On the other hand, the growth is maximized for a phase advance of $\phi=\pi / 4$, i.e. $n=h / 2$. For such a bucket (i.e. a bucket across the ring from the one into which beam is being injected) the relative growth of the invariant is maximized and expressed as $\Delta_{\max }=\cos 2 \pi v_{x}$. For the MAX IV $3 \mathrm{GeV}$ ring $\left(v_{x}=42.2\right)$ this amounts to a growth of $31 \%$ whereas for the MAX IV $1.5 \mathrm{GeV}$ ring and Solaris $\left(v_{x}=11.22\right)$ this growth is $19 \%$. Considering that the stored beam invariant scales with the kick $A_{\mathrm{st}}^{2} \propto \theta_{\mathrm{ki}}^{2}$, the upper limit for the injection kick $\theta_{\max }$ discussed in Section 2 needs to be reduced by the square root of the invariant increase derived here, i.e. by $13 \%(8 \%)$ in the $3 \mathrm{GeV}(1.5 \mathrm{GeV})$ storage ring.

Since the above calculations and considerations are based on a linear optics approximation, tracking studies through the actual lattice including sextupoles and octupoles are required to determine if accumulation efficiency is decreased by this choice of kicker pulse. DIMAD has been used to track stored beam particles in a bucket $n=h / 2$ in order to investigate the case where the stored beam invariant is maximized after receiving two kicks. Fig. A2 shows the results of these studies and can be directly compared to Figs. 7 and 15. Although the stored beam is excited to a larger invariant than in the case where it receives only a single kick, it appears the stored beam can still be contained within the storage ring acceptance in both cases. However, in the case of the $3 \mathrm{GeV}$ storage ring, the margin for error has been considerably reduced. A possible remedy is a reduction of dipole kick strength. This will decrease the stored beam invariant at the expense of the injected beam's reduced invariant. Since the latter is lower than the former, this trade-off should be beneficial in terms of accumulation efficiency. An example is displayed in Fig. A3 where a reduced kick of $\theta_{\mathrm{ki}}=-2.1 \mathrm{mrad}$ has been chosen. One should note that the stored beam particles shown here can again be considered a worst case since the chosen bucket results in maximum invariant.

While these studies indicate accumulation with a single dipole kicker should remain successful despite a half-sine pulse with $2 T_{\text {rev }}$ base length, they do not reveal the smearing out of the stored beam in phase space as a result of different buckets receiving different kicks. In order to give an estimate for this effect, DIMAD tracking studies were repeated using six different buckets at equal spacing to model the stored beam. Again, it was assumed injection takes place into bucket 0 . The results of these studies are displayed in Fig. A4. In addition to showing the smearing out of the stored beam, these results confirm that the largest invariant is reached by the $n=h / 2$ buckets.

Finally, it should be noted that for the Solaris case, the effect demonstrated here should be less severe because of the lower 

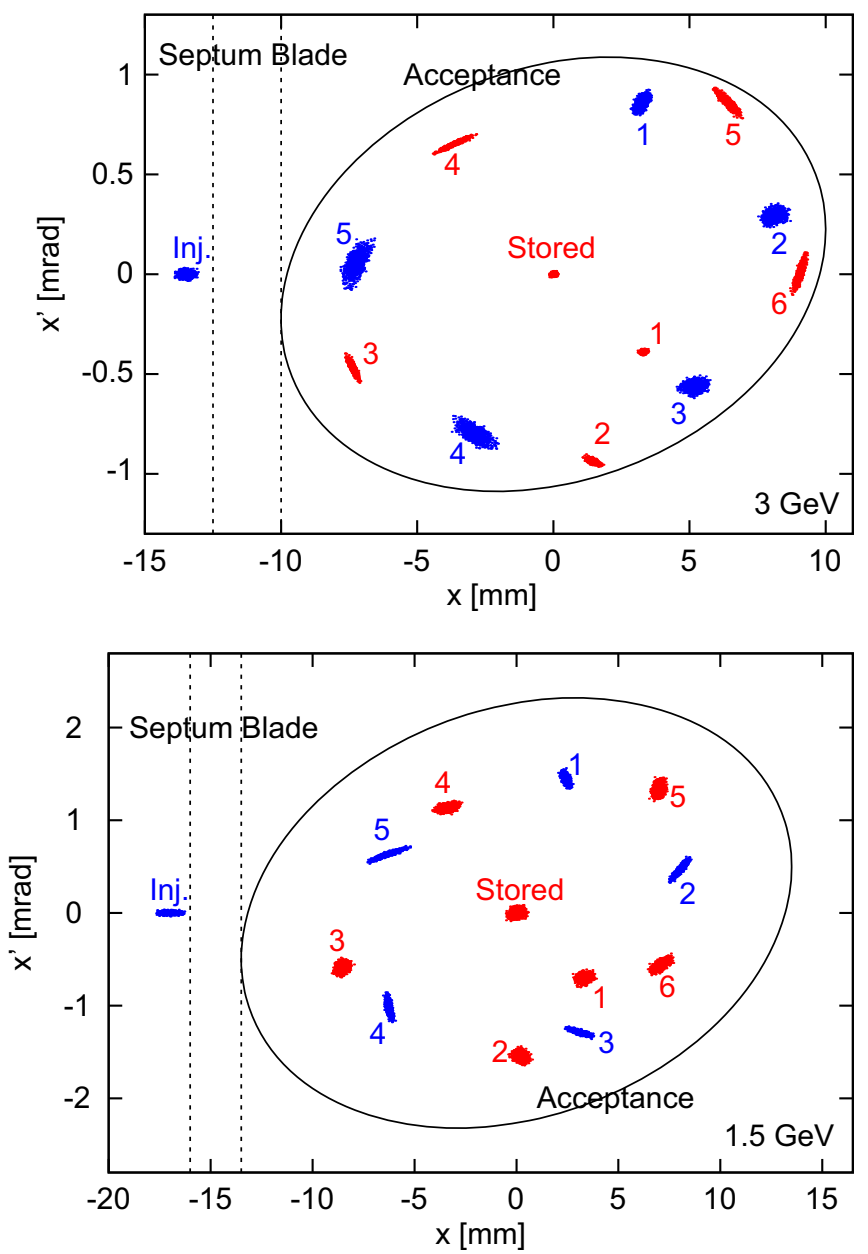

Fig. A2. DIMAD tracking showing accumulation of injected particles in the MAX IV $3 \mathrm{GeV}$ storage ring using $\theta_{\mathrm{ki}}=-2.3 \mathrm{mrad}$ (top) and $1.5 \mathrm{GeV}$ storage ring using $\theta_{\mathrm{ki}}=+1.4 \mathrm{mrad}$ (bottom). The stored beam particles shown here are $h / 2$ buckets away from bucket 0 into which injection is taking place. Therefore, stored beam particles receive a kick on two turns. The plots show injection, capture, and the first turns in the storage ring at the IP. These plots can be directly compared to the ideal cases shown in Figs. 7 and 15

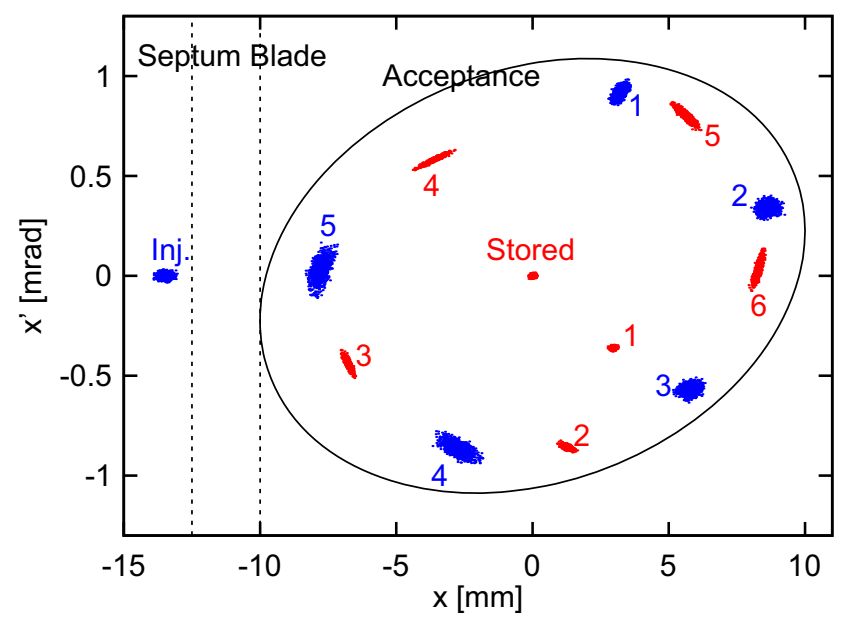

Fig. A3. DIMAD tracking showing accumulation of injected particles in the MAX IV $3 \mathrm{GeV}$ storage ring using $\theta_{\mathrm{ki}}=-2.1 \mathrm{mrad}$. The stored beam particles shown here are $h / 2$ buckets away from bucket 0 into which injection is taking place. The plot shows injection, capture, and the first turns in the storage ring at the IP. This plot can be directly compared to the case shown in Fig. A2 (top).
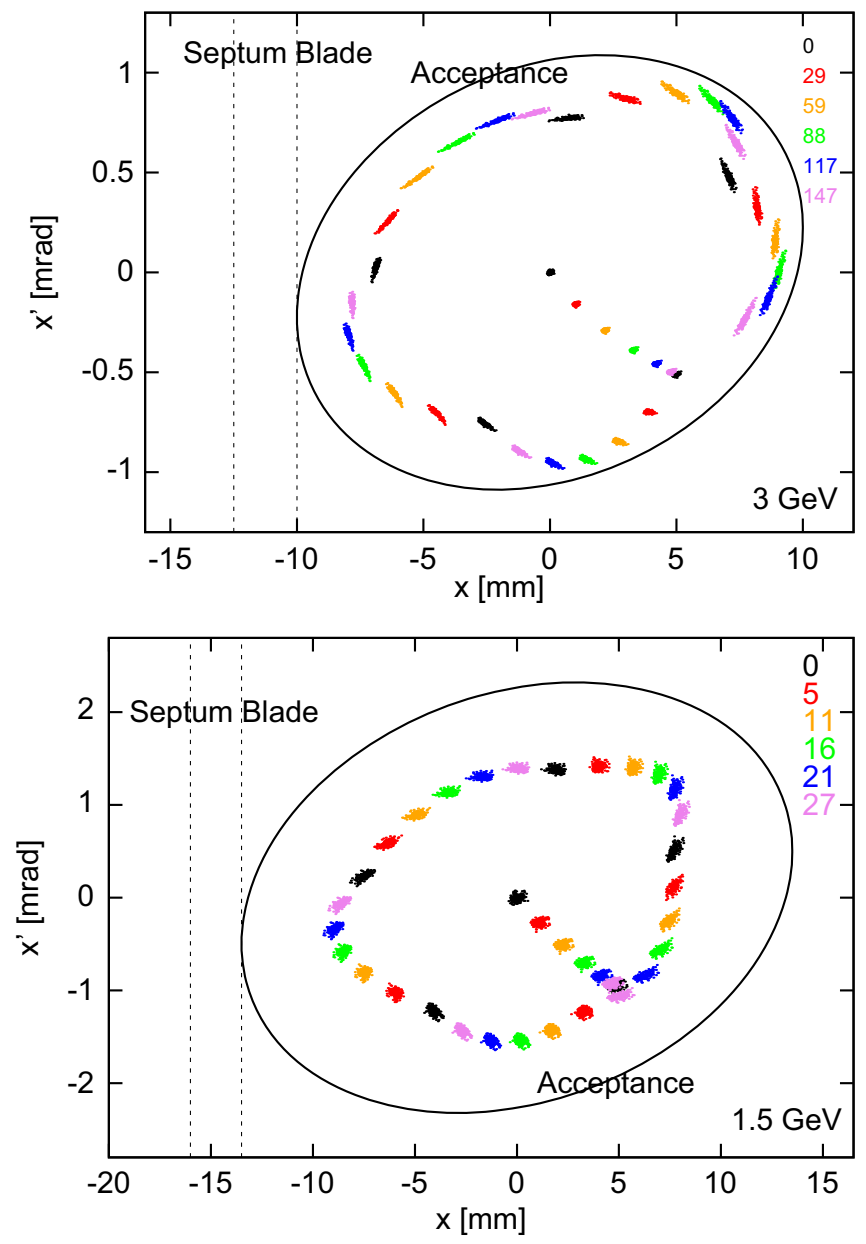

Fig. A4. DIMAD tracking at the IP showing the perturbation of stored beam particles in the MAX IV $3 \mathrm{GeV}$ storage ring when using the dipole kicker for injection with $\theta_{\mathrm{ki}}=-2.3 \mathrm{mrad}$ (top) and in the MAX IV $1.5 \mathrm{GeV}$ storage ring when using the dipole kicker for injection with $\theta_{\mathrm{ki}}=+1.4 \mathrm{mrad}$ (bottom). The stored beam particles shown here are color-coded according to bucket number (indicated at the top right) from bucket 0 (corresponding to the bucket into which injection is taking place) to bucket $h$. The different kicks received by different buckets lead to a smearing out of the stored beam. Bucket 0 corresponds to the stored beam particles shown in Figs. 7 and 15, whereas bucket $h / 2$ corresponds to the stored beam particles shown in Fig. A2. (For interpretation of the references to color in this figure legend, the reader is referred to the web version of this article.)

emittance of the stored beam. Although the stored beam invariant is increased for buckets away from the injection bucket, the smearing of the stored beam should be restricted to a smaller area as a result of the reduced stored beam emittance. Thus, accumulation efficiency should be slightly better than in the MAX IV $1.5 \mathrm{GeV}$ storage ring case.

\section{References}

[1] MAX IV Project 〈http://www.maxlab.lu.se/maxlab/max4〉.

[2] S.C. Leemann, et al., Physical Review Special Topics-Accelerators and Beams 12 (2009) 120701.

[3] S.C. Leemann, Updates to the MAX IV $3 \mathrm{GeV}$ Storage Ring Lattice, MAX-Lab Internal Note 20110117 〈http://www.maxlab.lu.se/maxlab/max4/max_iv_ reports_public $>$.

[4] S.C. Leemann, et al., Status of the MAX IV storage rings, in: Proceedings of IPAC10, WEPEA058, Kyoto, Japan, May 2010, p. 2618.

[5] S.C. Leemann, Updates to the MAX IV $1.5 \mathrm{GeV}$ Storage Ring Lattice, MAX-Lab Internal Note 20111102 <http://www.maxlab.lu.se/maxlab/max4/max_iv_ reports_public $>$.

[6] S. Thorin, et al., Design of the MAX IV Ring Injector and SPF/FEL Driver, in: Proceedings of the PAC'11, New York, USA, March 28-April 1, 2011, THP178. 
[7] MAX IV detailed design report 〈http://www.maxlab.lu.se/maxlab/max4/ DDR_public $>$.

[8] S.C. Leemann, Physical Review Special Topics-Accelerators and Beams 15 (2012) 050705.

[9] K. Harada, Y. Kobayashi, T. Miyajima, S. Nagahashi, Physical Review Special Topics-Accelerators and Beams 10 (2007) 123501.

[10] H. Takaki, et al., Physical Review Special Topics-Accelerators and Beams 13 (2010) 020705.

[11] S.C. Leemann, A. Streun, Physical Review Special Topics-Accelerators and Beams 14 (2011) 030701.

[12] S. Thorin, Main Linac Optic Modes, Internal Note, January 2011, Updated July 2011, unpublished.
[13] C. Bocchetta, et al., Project Status of the Polish Synchrotron Radiation Facility Solaris, in: Proceedings of the IPAC'11, San Sebastían, Spain, 2011, THPC054, p. 3014.

[14] A. Wawrzyniak, et al., Injector Layout and Beam Injection into Solaris, in: Proceedings of the IPAC'11, San Sebastían, Spain, 2011, THPC123, p. 3173.

[15] E. Al-dmour, et al., Vacuum system design for the MAX IV $3 \mathrm{GeV}$ ring, in: Proceedings of the IPAC'11, TUPS016, San Sebastían, Spain, 2011, p. 1554.

[16] MAX-Lab Internal Note, March 2011, Updated March 2012, unpublished.

[17] MAX-Lab Internal Note, February 2011, Updated February 2012, unpublished.

[18] Y. Cerenius, P. Sondhauss, Functional Description of the Front Ends on MAX IV, Internal MAX-lab Report, Version 2.2, March 2011. 